TRANSACTIONS OF THE

AMERICAN MATHEMATICAL SOCIETY

Volume 348, Number 12, December 1996, Pages 5087-5111

S 0002-9947(96)01779-5

\title{
LÉVY GROUP ACTION AND INVARIANT MEASURES ON $\beta \mathbb{N}$
}

\author{
MARTIN BLÜMLINGER
}

\begin{abstract}
For $f \in \ell^{\infty}(\mathbb{N})$ let $T f$ be defined by $T f(n)=\frac{1}{n} \sum_{i=1}^{n} f(i)$. We investigate permutations $g$ of $\mathbb{N}$, which satisfy $T f(n)-T f_{g}(n) \rightarrow 0$ as $n \rightarrow \infty$ with $f_{g}(n)=f(g n)$ for $f \in \ell^{\infty}(\mathbb{N})$ (i.e. $g$ is in the Lévy group $\mathcal{G}$ ), or for $f$ in the subspace of Cesàro-summable sequences. Our main interest are $\mathcal{G}$-invariant means on $\ell^{\infty}(\mathbb{N})$ or equivalently $\mathcal{G}$-invariant probability measures on $\beta \mathbb{N}$. We show that the adjoint $T^{*}$ of $T$ maps measures supported in $\beta \mathbb{N} \backslash \mathbb{N}$ onto a weak*-dense subset of the space of $\mathcal{G}$-invariant measures. We investigate the dynamical system $(\mathcal{G}, \beta \mathbb{N})$ and show that the support set of invariant measures on $\beta \mathbb{N}$ is the closure of the set of almost periodic points and the set of nontopologically transitive points in $\beta \mathbb{N} \backslash \mathbb{N}$. Finally we consider measures which are invariant under $T^{*}$.
\end{abstract}

\section{INTRODUCTION}

For $A \subset \mathbb{N}$ the density function is given by $d_{A}(n)=\frac{1}{n}|\{i \in A: i \leq n\}|$, and its upper density $\overline{d_{A}}$ as $\overline{d_{A}}=\overline{\lim }_{n \rightarrow \infty} d_{A}(n)$. A set $A$ has density $\alpha$ if $\lim _{n \rightarrow \infty} d_{A}(n)=$ $\alpha$. For a function $f \in \ell^{\infty}(\mathbb{N})$ (the space of bounded real functions on $\mathbb{N}$ ) we define the operator $T$ by $T f(n)=\frac{1}{n} \sum_{i=1}^{n} f(i)$.

Any element $f$ of $\ell^{\infty}(\mathbb{N})$ has a unique continuous extension to the Stone-Cech compactification $\beta \mathbb{N}$ of $\mathbb{N}$. The continuous extension of the characteristic functions $\chi_{A}$ of a subset $A$ of $\mathbb{N}$ is the characteristic function $\chi_{\bar{A}}$ of the closure $\bar{A}$ of $A$ in $\beta \mathbb{N}$. For a general $f$ in $\ell^{\infty}(\mathbb{N})$ we will denote the continuous extension of a function $f$ to $\beta \mathbb{N}$ again by $f$. This isometry between $\ell^{\infty}(\mathbb{N})$ and $C(\beta \mathbb{N})$ (the space of real continuous functions on $\beta \mathbb{N}$ ) allows us to express continuous functions on $\beta \mathbb{N}$ by their values on the integers, i.e. as bounded sequences on $\mathbb{N}$, to interpret $T$ as a continuous operator on $C(\beta \mathbb{N})$, and to identify the dual spaces of $\ell^{\infty}(\mathbb{N})$ (finitely additive measures on $\mathbb{N}$ ) and of $C(\beta \mathbb{N}$ ) (regular Borel measures on $\beta \mathbb{N}$ ). In particular means on $\ell^{\infty}(\mathbb{N})$ correspond to probability measures on $\beta \mathbb{N}$ (see proof of Lemma 1 below).

This correspondence between finitely additive measures on $\mathbb{N}$ and measures on $\beta \mathbb{N}$ becomes more transparent when we realize $\beta \mathbb{N}$ as the set of ultrafilters on $\mathbb{N}$, with ultrafilters being finitely additive 0-1 valued measures (cf. [11], 20.37 (b)): Then for an ultrafilter $q$ the Dirac measure $\delta_{q}$ is the 0-1 valued Borel measure on $\beta \mathbb{N}$ corresponding to the finitely additive $0-1$ valued measure $q$ on $\mathbb{N}$.

For our approach however it will be more convenient to work with the usual topological definition of a filter on a set $M$ as a nonempty collection $\mathfrak{F}$ of subsets of

Received by the editors September 29, 1995.

1991 Mathematics Subject Classification. Primary 54H20.

Part of this work was carried out at Macquarie University with financial support from the Australian Research Council.

(C)1996 American Mathematical Society 
$M$ satisfying (i) $\emptyset \notin \mathfrak{F}$, (ii) $A \in \mathfrak{F}, B \in \mathcal{F} \Rightarrow A \cap B \in \mathfrak{F}$, (iii) $A \in \mathfrak{F}, A \subset B \subset M \Rightarrow$ $B \in \mathfrak{F}$. An ultrafilter is a filter with the additional property that it is not properly contained in any other filter (i.e. it is maximal), or equivalently, $A \subset M \Rightarrow A \in \mathfrak{F}$ or $\complement A \in \mathfrak{F}$.

We identify the natural numbers with their neighborhood filters, so $\mathbb{N} \subset \beta \mathbb{N}$. A base for the topology on $\beta \mathbb{N}$ is now given by the clopen sets. A subset of $\beta \mathbb{N}$ is clopen iff it is the closure $\bar{A}$ of a subset $A$ of $\mathbb{N}$, i.e. iff it is the set of all ultrafilters containing $A$. With this abuse of notation we can see $A \subset \mathbb{N}$ both as a subset of $\beta \mathbb{N}$ and as an element of an ultrafilter $p$ in $\bar{A}$, i.e. we have $p \in \bar{A}$ iff $A \in p$. See [20] for a detailed exposition.

A permutation $g$ of $\mathbb{N}$ acts naturally as an automorphism on $\beta \mathbb{N}$ : For $p \in \beta \mathbb{N}$ let the ultrafilter $g p$ be defined as $g p=\{g A: A \in p\}$. So $g A \in g p \Leftrightarrow A \in p$ and $g_{1}\left(g_{2} p\right)=\left(g_{1} g_{2}\right) p$, i.e. the group of permutations of $\mathbb{N}$ acts as a transformation group on $\beta \mathbb{N}$. Then for any clopen set $\bar{A}, A \subset \mathbb{N}$, we have

$$
g \bar{A}=\{g p: p \in \bar{A}\}=\{g p: A \in p\}=\{g p: g A \in g p\}=\{q: g A \in q\}=\overline{g A} .
$$

Since the clopen sets are a base for the topology on $\beta \mathbb{N}$, it follows that $g$ is an automorphism.

A permutation $g$ of $\mathbb{N}$ also induces an isometry of $\ell^{\infty}(\mathbb{N}): f \rightarrow f_{g}, f_{g}(n)=f(g n)$ and an isometry of $C(\beta \mathbb{N}): f \rightarrow f_{g}, f_{g}(p)=f(g p), p \in \beta \mathbb{N}$, so $g$ acts naturally on the space $\mathcal{M}$ of (signed real regular Borel) measures on $\beta \mathbb{N}$ by $\langle g \mu, f\rangle=\left\langle\mu, f_{g}\right\rangle$ or equivalently by $g \mu(U)=\mu\left(g^{-1} U\right)$ for Borel sets $U$ in $\beta \mathbb{N}$. A measure $\mu$ in $\mathcal{M}$ is $G$-invariant ( $G$ being some group of permutations) if $\mu=\mu_{g} \forall g \in G$, i.e. if $\mu$ is in the annihilator $\left\{f-f_{g}: f \in C(\beta \mathbb{N}), g \in G\right\}^{\perp}$ (with respect to the duality $\langle\mathcal{M}, C(\beta \mathbb{N})\rangle)$.

We shall be interested in measures on $\beta \mathbb{N}$, which are invariant under certain permutations defined via invariance of density functions at infinity. The Lévy group $\mathcal{G}$ is the group of permutations $g$ satisfying $T f-T f_{g}$ vanishes on $\mathbb{N}^{*} \forall f \in C(\beta \mathbb{N})$. We construct a weak*-dense subspace of the space of $\mathcal{G}$-invariant measures and show that the bigger group $\mathcal{G}_{\delta}$ of permutations satisfying this requirement only for Cesáro-summable bounded sequences $f$ allows no $\mathcal{G}_{\delta}$-invariant measures.

We investigate the topological dynamics of the permutation group $\mathcal{G}$ acting on $\beta \mathbb{N}$; similar problems of invariance under right translations (or more generally under motions, i.e. 1-1 maps of $\mathbb{N}$ without periodic points) were discussed in [4], [12], [16] and [17]. See [2], II.5 for an overview.

Although right translation $\tau$ of $\mathbb{N}$ is not a permutation of $\mathbb{N}$, there are elements $g$ in $\mathcal{G}$ which satisfy $g n=\tau n$ except for a set of density 0 (cf. proof of Theorem 7 ). Since sets of density 0 will turn out to be negligible for the questions considered, it is safe to think of translations as being a subsemigroup of the Lévy group. In particular the action of $\tau$ on the support set $S$ of measures invariant under the Lévy group coincides with the action of certain elements of the Lévy group on $S$.

While the characterization of the support set $S_{\tau}$ of $\tau$-invariant measures looks like our characterization of $S$ (Theorem 4 ) with the notion of upper density replaced by that of upper Banach density, the orbit structures of the pertinent dynamical systems differ drastically: While we will show that points with minimal $\mathcal{G}$-orbit closure are dense in $S$, it is known that there are open sets in $S_{\tau}$ without points with minimal $\tau$-orbit closure. On the other hand every minimal $\tau$-orbit closure is the support set of some $\tau$-invariant measure, whereas there are minimal $\mathcal{G}$-orbit closures which do not support any $\mathcal{G}$-invariant measure. 
Some of these differences are due to the fact that the Lévy group is not amenable, whereas the semigroup of translations is amenable. An essential difference in the investigations of the dynamical systems $(\mathcal{G}, \beta \mathbb{N})$ and $(\tau, \beta \mathbb{N})$ stems from the fact that translations of $\mathbb{N}$ suggest the consideration of $\mathbb{N}$ as a semigroup under addition, which then allows some characterizations via algebraic properties of the left topological (right topological in the notation of [2]) semigroup $\beta \mathbb{N}$. So the set $S$ is a right ideal of the semigroup $(\beta \mathbb{N},+)$ cf. [5], 7.4 and minimal closed right ideals are just minimal orbit closures under $\tau$ cf. [1], 2.1.

Finally we characterize the support set of measures which are invariant under the adjoint $T^{*}$ of $T$. This invariance is a stronger requirement than $\mathcal{G}$-invariance. The support set $S_{I}$ of these measures allows a characterization similar to that of $S$ or of $S_{I}$, again with an appropriate notion of density.

\section{PERMUtATion GRoups AND INVARIANT MEANS ON $\mathbb{N}$}

We first clarify why the existence of positive invariant functionals is equivalent to the existence of nonzero invariant functionals for $\ell^{\infty}(\mathbb{N})$ resp. $C(\beta \mathbb{N})$. The proof of these facts is included for the reader's convenience.

We recall that a mean on $\mathbb{N}$ is a positive normalized finitely additive measure (set function) on $\mathbb{N}$ or equivalently a positive normalized functional on $\ell^{\infty}(\mathbb{N})$.

Lemma 1. For a group $G$ of permutations of $\mathbb{N}$ the following are equivalent:

(a) There exists a $G$-invariant mean on $\mathbb{N}$;

(b) There exists a nonzero $G$-invariant finitely additive measure on $\beta \mathbb{N}$;

(c) There exists a $G$-invariant probability measure on $\beta \mathbb{N}$;

(d) There exists a nonzero $G$-invariant (finite Borel) measure on $\beta \mathbb{N}$.

Proof. The restriction map from $\beta \mathbb{N}$ to $\mathbb{N}$ induces an isometry $\Phi$ between the Banach spaces $C(\beta \mathbb{N})$ and $\ell^{\infty}(\mathbb{N})$. The adjoint $\Phi^{*}$ then is an isometry between their dual spaces: $\Phi^{*}: \ell^{\infty}(\mathbb{N})^{*} \rightarrow C(\beta \mathbb{N})^{*}$. The dual of $\ell^{\infty}(\mathbb{N})$ is the space of finitely additive measures on $\mathbb{N}$ (cf. [6], IV 8.16) and the dual of $C(\beta \mathbb{N}$ ) is the space $\mathcal{M}$ of (finite real regular Borel) measures on $\beta \mathbb{N}$ by the Riesz representation theorem (cf. [6], IV 6.2), both spaces with the total variation norm.

We further note that, since for $f \in C(\beta \mathbb{N})$ and a permutation $g$ of $\mathbb{N}$ the action of $g$ on $f$ is defined by $f_{g}(p)=f(g p) \forall p \in \beta \mathbb{N}$, we have

$$
(\Phi f)_{g}(n)=(\Phi f)(g n)=f(g n)=f_{g}(n)=\Phi\left(f_{g}\right)(n),
$$

i.e. the action of $G$ commutes with the isometry $\Phi$ and we may use the notation $f_{g}$ both for the continuous extension of $f_{g} \in \ell^{\infty}(\mathbb{N})$ to $\beta \mathbb{N}$ and for the action of $g$ on the continuous extension of $f \in \ell^{\infty}(\mathbb{N})$ to $\beta \mathbb{N}$.

Since the space of $G$-invariant measures on $\beta \mathbb{N}$ is just the annihilator of the set $\left\{f-f_{g}: f \in C(\beta \mathbb{N}), g \in G\right\}$ and the space of $G$-invariant finitely additive measures on $\mathbb{N}$ is just the annihilator of the set $\left\{f-f_{g}: f \in \ell^{\infty}(\mathbb{N}), g \in G\right\}$, it follows that $G$-invariant measures on $\beta \mathbb{N}$ correspond to $G$-invariant finitely additive measures on $\mathbb{N}$ via the isometry $\Phi^{*}$, since by (2) $\Phi$ is a bijection between the above two sets.

Finally $\Phi$ is order preserving since the restriction to $\mathbb{N}$ of a positive function on $C(\beta \mathbb{N})$ is positive, so means correspond to probability measures and (a) $\Leftrightarrow$ (c) and (b) $\Leftrightarrow$ (d) follow.

Since (c) $\Rightarrow$ (d) is trivial, it remains to show (d) $\Rightarrow(\mathrm{c})$ : Let $\mu$ be a nontrivial $G$ invariant measure on $\beta \mathbb{N}$ and let $\beta \mathbb{N}=X_{1} \cup X_{2}$ be the Hahn decomposition (cf. [11]) of $\beta \mathbb{N}$, i.e. $\mu$ is nonnegative when restricted to $X_{1}$ and nonpositive when restricted 
to $X_{2}$, where $X_{1}, X_{2}$ are disjoint Borel subsets of $\beta \mathbb{N}$. Assume $\mu$ is nonzero on $X_{1}$ (otherwise $-\mu$ is positive and invariant) and let $\mu_{+}$be the positive part of $\mu$, i.e. $\mu_{+}(E)=\mu\left(E \cap X_{1}\right)$ for Borel sets $E$ in $\beta \mathbb{N}$.

For $g \in G$ we obtain using the $G$-invariance of $\mu$ :

$$
0 \leq \mu_{+}\left(g^{-1} X_{2}\right)=\mu\left(g^{-1} X_{2} \cap X_{1}\right)=\mu\left(X_{2} \cap g X_{1}\right) \leq 0,
$$

hence $\mu_{+}\left(g^{-1} X_{2}\right)=0$. The same argument applies to $g^{-1}$, so $\mu_{+}\left(g X_{2}\right)=0$ and it follows that $\mu_{+}\left(g X_{1}\right)=\mu_{+}\left(g^{-1} X_{1}\right)=\mu_{+}(\beta \mathbb{N})$. Thus for any Borel subset $E$ of $\beta \mathbb{N}$ we get

$$
\begin{aligned}
\mu_{+}(E) & =\mu_{+}\left(E \cap g^{-1} X_{1}\right)=\mu\left(E \cap g^{-1} X_{1} \cap X_{1}\right) \\
& =\mu\left(g E \cap X_{1} \cap g X_{1}\right)=\mu_{+}\left(g E \cap g X_{1}\right)=\mu_{+}(g E) .
\end{aligned}
$$

So $\mu_{+}$is $G$-invariant. Since $\mu_{+}$is positive and nonzero $\frac{1}{\left\|\mu_{+}\right\|} \mu_{+}$is a $G$-invariant probability measure.

There are two natural permutation groups characterized by invariance of densities of natural numbers cf. [13], [14], [15], [3]:

Let $\mathcal{G}_{\delta}$ be the group of permutations $g$ of $\mathbb{N}$ satisfying

$$
\lim _{n \rightarrow \infty} d_{A}(n)=\lim _{n \rightarrow \infty} d_{g A}(n)=\lim _{n \rightarrow \infty} d_{g^{-1} A}(n)
$$

for all subsets $A$ of $\mathbb{N}$ which have a density, i.e. for which $\lim _{n \rightarrow \infty} d_{A}(n)$ exists. By [3], Theorem 1 an equivalent definition of $\mathcal{G}_{\delta}$ is given as the group of permutations $g$ for which

$$
\lim _{n \rightarrow \infty} T f(n)=\lim _{n \rightarrow \infty} T f_{g}(n)=\lim _{n \rightarrow \infty} T f_{g^{-1}}(n)
$$

for all $f$ in the subspace of Cesàro-summable sequences in $\ell^{\infty}(\mathbb{N})$.

This group contains the proper subgroup of permutations $g$ satisfying

$$
\lim _{n \rightarrow \infty} d_{A}(n)-d_{g A}(n)=0 \quad \forall A \subset \mathbb{N} .
$$

The Lévy group $\mathcal{G}$ (first considered in [13], 3.36) is defined as the group of permutations $g$ of $\mathbb{N}$, for which

$$
\lim _{N \rightarrow \infty} \frac{1}{N}|\{n: n \leq N, g n>N\}|=0 .
$$

The following lemma identifies the Lévy group with the above subgroup of $\mathcal{G}_{\delta}$ :

Lemma 2. The following are equivalent:

$$
\begin{aligned}
& \text { (a) } g \in \mathcal{G} ; \\
& \text { (b) } \forall f \in \ell^{\infty}(\mathbb{N}) \quad T f(n)-T f_{g}(n) \underset{n \rightarrow \infty}{\longrightarrow} 0 ; \\
& \text { (c) } \forall A \subset \mathbb{N} \quad d_{A}(n)-d_{g A}(n) \underset{n \rightarrow \infty}{\longrightarrow} 0 .
\end{aligned}
$$

Proof. The definition of $\mathcal{G}$ shows that (a) implies (b). Clearly (b) implies (c) since $d_{A}(n)=T \chi_{A}(n)$.

If $g \notin \mathcal{G}$, then there exists a sequence $\left(n_{i}\right)$ with $n_{i-1} / n_{i} \rightarrow 0$ and $0<\alpha \in \mathbb{R}$, such that the sets $E_{i}:=\left\{n: n \leq n_{i}, g n>n_{i}\right\}$ satisfy $\left|E_{i}\right| / n_{i}>\alpha$. For $A:=\bigcup_{i} E_{i}$ we obtain $d_{A}\left(n_{i}\right)>d_{E_{i}}\left(n_{i}\right)>\alpha$. Since $\left.g E_{j} \subset\right] n_{j}, \infty[$ we obtain

$$
d_{g A}\left(n_{i}\right) \leq d_{\cup_{j<i} E_{j}}\left(n_{i}\right) \leq \frac{n_{i-1}}{n_{i}} \underset{i \rightarrow \infty}{\longrightarrow} 0
$$

and $\overline{\lim }_{i \rightarrow \infty} d_{A}\left(n_{i}\right)-d_{g A}\left(n_{i}\right) \geq \alpha>0$, so (c) implies (a). 
We will also need the following generalization of [15], Lemma 2.4:

Lemma 3. Let $A, B \subset \mathbb{N}$ such that $A, B, \complement A, \complement B$ are infinite sets. Then there is a $g \in \mathcal{G}$ with $B=g A$ if and only if $\lim _{n \rightarrow \infty} d_{A}(n)-d_{B}(n)=0$.

Proof. Let $A=\left\{a_{1}<a_{2}<\ldots\right\}, \complement A=\left\{a_{1}^{\prime}<a_{2}^{\prime}<\ldots\right\}$ and $B=\left\{b_{1}<b_{2}<\ldots\right\}$, $\complement B=\left\{b_{1}^{\prime}<b_{2}^{\prime}<\ldots\right\}$. We define the permutation $g$ by $g a_{i}=b_{i}, g a_{i}^{\prime}=b_{i}^{\prime}$. We have

$$
\begin{aligned}
\frac{1}{N} \mid\{n & : n \leq N, g n>N\} \mid \\
& =\frac{1}{N}|\{n \in A: n \leq N, g n>N\}|+\frac{1}{N} \mid\{n \in \complement[A: n \leq N, g n>N\} \mid \\
& =\max \left(0, d_{A}(N)-d_{B}(N)\right)+\max \left(0, d_{\complement_{A}}(N)-d_{\mathfrak{C}_{B}}(N)\right) .
\end{aligned}
$$

If $\lim _{n \rightarrow \infty} d_{A}(n)-d_{B}(n)=0$, it follows that $\lim _{n \rightarrow \infty} d_{\mathfrak{C} A}(n)-d_{\mathfrak{C} B}(n)=0$, and we see that $\lim _{N \rightarrow \infty} \frac{1}{N}|\{n: n \leq N, g n>N\}|=0$, i.e. $g \in \mathcal{G}$.

The "only if" statement follows from Lemma 2 above.

We now show that the group $\mathcal{G}_{\delta}$ does not admit an invariant mean on $\ell^{\infty}(\mathbb{N})$. Since we are mainly interested in invariant measures this explains why we have to restrict our considerations concerning invariant measures to the Lévy group $\mathcal{G}$.

Theorem 1. There exists no nontrivial $\mathcal{G}_{\delta}$-invariant functional on $\ell^{\infty}(\mathbb{N})$.

Proof. For $a+l \leq b, \quad a, b, l \in \mathbb{N}$ let $g(a, a+l, b, b+l)$ be the permutation

$$
x \mapsto \begin{cases}x+b-a & \text { for } x \in[a, a+l[, \\ x-(b-a) & \text { for } x \in[b, b+l[ \\ x & \text { else, }\end{cases}
$$

that is $g(a, a+l, b, b+l)$ transposes the intervals of integers $[a, a+l[$ and $[b, b+l[$. We define permutations $g_{1}, \ldots, g_{4}$ as the product of pairwise disjoint transpositions:

$$
\begin{aligned}
& g_{1}=\prod_{i \geq 0} g\left(2^{2 i}, 2^{2 i+1}, 2^{2 i+1}, 2^{2 i+1}+2^{2 i}\right), \\
& g_{2}=\prod_{i \geq 0} g\left(2^{2 i+1}, 2^{2 i+2}, 2^{2 i+2}, 2^{2 i+2}+2^{2 i+1}\right), \\
& g_{3}=\prod_{i \geq 0} g\left(2^{2 i}, 2^{2 i+1}, 2^{2 i+1}+2^{2 i}, 2^{2 i+2}\right), \\
& g_{4}=\prod_{i \geq 0} g\left(2^{2 i+1}, 2^{2 i+2}, 2^{2 i+2}+2^{2 i+1}, 2^{2 i+3}\right) .
\end{aligned}
$$

An easy computation, or combining [3], Theorem 1 and [14], proof of Proposition 4.1 , p. 106, shows that the permutation $\prod_{i} g\left(a_{i}, a_{i}+l_{i}, a_{i}+l_{i}, a_{i}+2 l_{i}\right)$ is in $\mathcal{G}_{\delta}$ for $a_{i}+2 l_{i} \leq a_{i+1}$. Therefore $g_{1}, g_{2} \in \mathcal{G}_{\delta}$. Since

$$
\begin{aligned}
g_{3}=h_{1} h_{2} h_{1} & =g\left(2^{2 i}, 2^{2 i+1}, 2^{2 i+1}+2^{2 i}, 2^{2 i+2}\right) \quad \text { with } \\
h_{1} & =g\left(2^{2 i}, 2^{2 i+1}, 2^{2 i+1}, 2^{2 i+1}+2^{2 i}\right) \\
h_{2} & =g\left(2^{2 i+1}, 2^{2 i+1}+2^{2 i}, 2^{2 i+1}+2^{2 i}, 2^{2 i+2}\right)
\end{aligned}
$$

it follows that $g_{3} \in \mathcal{G}_{\delta} . g_{4} \in \mathcal{G}_{\delta}$ can be seen similarly. Note that $\mathcal{G}_{\delta}$ as defined in [14] is a semigroup, however since $g_{i}=g_{i}^{-1}$, we have $g_{i} \in \mathcal{G}_{\delta}$. 
We define the functions $f_{i}, i=1,2,3,4$ by

$$
f_{i}(l)= \begin{cases}1, & g_{i} l>l, \\ 0, & \text { otherwise }\end{cases}
$$

It is easily verified that

$$
\left(f_{i}-\left(f_{i}\right)_{g_{i}}\right)(l)= \begin{cases}1, & g_{i} l>l, \\ -1, & g_{i} l<l, \\ 0, & g_{i} l=l .\end{cases}
$$

Hence for $y:=\sum_{i=1}^{4} f_{i}-\left(f_{i}\right)_{g_{i}}$ we have $y(l)=1$ for $l>1$ and $y(1)=2$.

The space of $\mathcal{G}_{\delta}$-invariant measures on $\beta \mathbb{N}$ can be considered as the annihilator $\left\{f-f_{g}: g \in \mathcal{G}_{\delta}, f \in C(\beta \mathbb{N})\right\}^{\perp}$ with respect to the duality $\langle\mathcal{M}, C(\beta \mathbb{N})\rangle$. Therefore $\mathcal{G}_{\delta}$-invariant measures vanish on $y$. Obviously any $\mathcal{G}_{\delta}$-invariant functional has to vanish on $c_{0}(\mathbb{N})$ since the dual of $c_{0}(\mathbb{N})$ can be identified with $\ell^{1}(\mathbb{N})$ and 0 is the only $\mathcal{G}_{\delta}$-invariant function in $\ell^{1}(\mathbb{N})$. In particular invariant measures vanish on the function $e_{1}: e_{1}(1)=1, e_{1}(n)=0$ for $n>1$. Since $y-e_{1}$ is just the constant function 1 , we see that any $\mathcal{G}_{\delta}$-invariant measure vanishes on constant functions. Since a positive normalized functional cannot vanish on constant functions we deduce that no mean on $\ell^{\infty}(\mathbb{N})$ is $\mathcal{G}_{\delta}$-invariant. By Lemma 1 this implies that no nontrivial functional on $\ell^{\infty}(\mathbb{N})$ (nontrivial finitely additive measure on $\mathbb{N}$ ) is $\mathcal{G}_{\delta}$-invariant.

We now give an explicit description of a weak*-dense subset of $\mathcal{G}$-invariant measures on $\beta \mathbb{N}$ corresponding to finitely additive $\mathcal{G}$-invariant set functions on $\mathbb{N}$. We remark that a similar characterization of invariant means for the translation semigroup was obtained in [12].

We denote the space of measures supported in $\mathbb{N}^{*}$ by $\mathcal{M}_{\infty}$ and the range of the restriction of the adjoint $T^{*}$ of $T$ to $\mathcal{M}_{\infty}$ by $\mathcal{R}$, i.e. $\mathcal{R}:=\left\{T^{*} \mu: \mu \in \mathcal{M}_{\infty}\right\} . \mathcal{M}_{\mathcal{G}}$ is the space of $\mathcal{G}$-invariant probability measures on $\beta \mathbb{N}$.

Since $\lim _{n \rightarrow \infty} T f(n)-T f_{g}(n)=0$ for $g \in \mathcal{G}$ by Lemma 2, it follows that $T f(x)=T f_{g}(x) \forall x \in \mathbb{N}^{*}, g \in \mathcal{G}$. Therefore the adjoint $T^{*}$ maps measures which are supported in $\mathbb{N}^{*}$ to the space of $\mathcal{G}$-invariant measures on $\beta \mathbb{N}$, i.e.

$$
\mathcal{R} \subseteq \mathcal{M}_{\mathcal{G}}
$$

and $T^{*}$ maps probability measures supported on $\mathbb{N}^{*}$ to $\mathcal{G}$-invariant probability measures.

The following counterexample shows that this map is not surjective, i.e. there are $\mathcal{G}$-invariant probability measures which are not the image under $T^{*}$ of a probability measure. Since this image is a weak*-compact set it follows that this image is not even dense.

For an ultrafilter $q$ the ultrafilter $2 q$ is defined by the filter base $\{2 A: A \in q\}$. Let $q \in \mathbb{N}^{*}$ be an ultrafilter containing the set $\left\{2^{2^{i}}: i \in \mathbb{N}\right\}$. For any positive function $f$ on $\mathbb{N}$ with $\|f\|=1$ we have

$$
\frac{1}{2} T f(n) \leq T f(2 n) \leq \frac{1}{2}(1+T f(n)) .
$$

Therefore

$$
\frac{1}{2}\left\langle\delta_{q}, T f\right\rangle \leq\left\langle\delta_{2 q}, T f\right\rangle \leq \frac{1}{2}\left(1+\left\langle\delta_{q}, T f\right\rangle\right)
$$


and

$$
1 \geq\left\langle T^{*}\left(2 \delta_{2 q}-\delta_{q}\right), f\right\rangle=\left\langle 2 \delta_{2 q}-\delta_{q}, T f\right\rangle \geq 0,
$$

so $T^{*}\left(2 \delta_{2 q}-\delta_{q}\right)$ is a probability measure as $\left\langle T^{*}\left(2 \delta_{2 q}-\delta_{q}\right), 1\right\rangle=1$. For the set $A=\bigcup_{i}\left[2^{2^{i}}, 2 \cdot 2^{2^{i}}\right.$ [ we obtain $\varlimsup_{n \rightarrow \infty} T \chi_{A}(n)=\lim _{i \rightarrow \infty} T \chi_{A}\left(2 \cdot 2^{2^{i}}\right)=1 / 2$, and $\lim _{i \rightarrow \infty} T \chi_{A}\left(2^{2^{i}}\right)=0$. Therefore $\left\langle\mu, T \chi_{\bar{A}}\right\rangle=\left\langle T^{*} \mu, \chi_{\bar{A}}\right\rangle \leq 1 / 2$ for any probability measure $\mu$ on $\mathbb{N}^{*}$ and $\left\langle 2 \delta_{2 q}-\delta_{q}, T \chi_{\bar{A}}\right\rangle=1$, so $\left\|T^{*} \mu-T^{*}\left(2 \delta_{2 q}-\delta_{q}\right)\right\| \geq 1 / 2$ for all probability measures $\mu$ supported in $\mathbb{N}^{*}$. Hence the probability measure $T^{*}\left(2 \delta_{2 q}-\delta_{q}\right)$ is not in the image of probability measures supported in $\mathbb{N}^{*}$ under the map $T^{*}$.

Theorem 2. $\mathcal{R}$ is a proper weak ${ }^{*}$-dense subset of the weak ${ }^{*}$-closed space $\mathcal{M}_{\mathcal{G}}$. The annihilator of the subspace $\mathcal{M}_{\mathcal{G}}$ with respect to the duality $\langle\mathcal{M}, C(\beta \mathbb{N})\rangle$ is the space $\left\{f \in C(\beta \mathbb{N}): T f(p)=0 \forall p \in \mathbb{N}^{*}\right\}$.

Proof. Clearly the annihilator $\mathcal{R}^{\perp}$ of $\mathcal{R}$ is $\left\{f \in C(\beta \mathbb{N}): T f(p)=0 \forall p \in \mathbb{N}^{*}\right\}=: \mathcal{L}_{0}$. By [3], Theorem 2 any $\mathcal{G}$-invariant functional on $\ell^{\infty}(\mathbb{N})$ is a multiple of Cesàro-mean on the subspace of Cesàro-summable functions, so $\mathcal{M}_{\mathcal{G}} \subseteq \mathcal{L}_{0}^{\perp}$. Since

$$
\mathcal{M}_{\mathcal{G}}=\left\{f-f_{g}: f \in C(\beta \mathbb{N}), g \in \mathcal{G}\right\}^{\perp}
$$

it follows that $\mathcal{M}_{\mathcal{G}}$ is weak*-closed. With (4) and the bipolar theorem cf. [18], III.7.4 it follows that $\mathcal{R}^{\perp \perp} \subseteq \mathcal{M}_{\mathcal{G}}$. Therefore

$$
\mathcal{M}_{\mathcal{G}} \supseteq \mathcal{R}^{\perp \perp}=\mathcal{L}_{0}^{\perp} \supseteq \mathcal{M}_{\mathcal{G}} \quad \text { and } \quad \mathcal{R}^{\perp \perp}=\mathcal{M}_{\mathcal{G}} .
$$

The bipolar theorem now also gives the weak*-density of $\mathcal{R}$ in $\mathcal{M}_{\mathcal{G}}$.

We next show that $\mathcal{R}$ is a proper subset of $\mathcal{M}_{\mathcal{G}}$. Let $C_{0}(\beta \mathbb{N})$ be the closed subspace of functions in $C(\beta \mathbb{N})$ which vanish on $\mathbb{N}^{*}$. We first note that

$$
\left(C(\beta \mathbb{N}) / C_{0}(\beta \mathbb{N})\right)^{*} \cong C_{0}(\beta \mathbb{N})^{\perp}=\mathcal{M}_{\infty}
$$

(cf. [6], II.4.18) and that $T$ maps $C_{0}(\beta \mathbb{N})$ to $C_{0}(\beta \mathbb{N})$, so $T$ induces a continuous map $\tilde{T}: \quad C(\beta \mathbb{N}) / C_{0}(\beta \mathbb{N}) \mapsto C(\beta \mathbb{N}) / C_{0}(\beta \mathbb{N})$. We can therefore identify the restriction of $T^{*}$ to $\mathcal{M}_{\infty}$ with the adjoint of $\tilde{T}$.

Since $\mathcal{M}_{\mathcal{G}}$ being weak*-closed is clearly norm-closed, it follows by the closed range theorem cf. [11], VI 6.4, that the adjoint of $\tilde{T}$ has no norm-closed range if $\tilde{T}$ has no norm-closed range. To see that $\mathcal{R} \neq \mathcal{M}_{\mathcal{G}}$, it therefore remains to show that the range of $\tilde{T}$ is not norm-closed.

For $\alpha>1$ let

$$
A_{\alpha}=\bigcup_{n \in \mathbb{N}}\left[2^{2^{n}}+1,\left[2^{2^{n}}(1+1 / \alpha)\right]\right]
$$

where $[m, n]$ is the interval $\{m, m+1, \ldots, n\}$ of integers and $[x]$ denotes the integer part of $x$, i.e. $[x]=\max \{n: n \in \mathbb{N}, n \leq x\}$ for $x \geq 1$. For $\psi_{\alpha}=\alpha \chi_{A_{\alpha}}$ we have $\lim _{i \rightarrow \infty} T \psi_{\alpha}\left(2^{2^{i}}\right)=0$ and

$$
\lim _{i \rightarrow \infty} T \psi_{\alpha}\left(\left[2^{2^{i}}(1+1 / \alpha)\right]\right)=\varlimsup_{n \rightarrow \infty} T \psi_{\alpha}(n)=\frac{\alpha}{1+\alpha} .
$$


Therefore any function $\hat{\psi}_{\alpha}$ on $\mathbb{N}$ with $\lim _{n \rightarrow \infty}\left(T\left(\psi_{\alpha}-\hat{\psi}_{\alpha}\right)\right)(n)=0$ satisfies

$$
\begin{gathered}
\varlimsup_{i \rightarrow \infty} \sup \left\{\hat{\psi}_{\alpha}(n): n \in\left[2^{2^{i}}+1,\left[2^{2^{i}}(1+1 / \alpha)\right]\right]\right\} \geq \varlimsup_{i \rightarrow \infty} \frac{1}{\left[2^{2^{i}} / \alpha\right]} \sum_{n=2^{2^{i}}+1}^{\left[2^{2^{i}}(1+1 / \alpha)\right]} \hat{\psi}_{\alpha}(n) \\
=\varlimsup_{i \rightarrow \infty} \frac{1+1 / \alpha}{1 / \alpha} T \hat{\psi}_{\alpha}\left(\left[2^{2^{i}}(1+1 / \alpha)\right]\right)-\frac{1}{1 / \alpha} T \hat{\psi}_{\alpha}\left(2^{2^{i}}\right)=(1+\alpha) \frac{\alpha}{1+\alpha}=\alpha .
\end{gathered}
$$

Hence any element $\tilde{\psi}_{\alpha}$ in the quotient space $C(\beta \mathbb{N}) / C_{0}(\beta \mathbb{N})$ with $\tilde{T} \tilde{\psi}_{\alpha}=T \psi_{\alpha}+$ $C_{0}(\beta \mathbb{N})$ satisfies $\left\|\tilde{\psi}_{\alpha}\right\|_{C(\beta \mathbb{N}) / C_{0}(\beta \mathbb{N})} \geq \alpha$. It follows that $\tilde{T}$ is not an open mapping since $\alpha$ is arbitrary but $\left\|\tilde{T} \tilde{\psi}_{\alpha}\right\|_{C(\beta \mathbb{N}) / C_{0}(\beta \mathbb{N})}=1$. So by the open mapping theorem the range of $\tilde{T}$ cannot be closed in $C(\beta \mathbb{N}) / C_{0}(\beta \mathbb{N})$.

\section{TOPOLOGICAL DYNAMICS OF $(\mathcal{G}, \beta \mathbb{N})$}

We start with a definition that will be useful in considering $\mathcal{G}$-invariant subsets of $\mathbb{N}^{*}$ :

A subset $A$ of $\mathbb{N}$ is almost invariant if the symmetric difference $A \triangle g A$ is a set of density 0 for all $g \in \mathcal{G}$.

A function $f \in C(\beta \mathbb{N})$ (resp. in $\ell^{\infty}(\mathbb{N})$ ) is almost invariant if for all $\epsilon>0$ and $g \in \mathcal{G}$ the set $\{n \in \mathbb{N}:|f(n)-f(g n)|>\epsilon\}$ is of density 0 . Clearly $A \subset \mathbb{N}$ is almost invariant iff the characteristic function $\chi_{A}$ is almost invariant.

The easiest nontrivial example of an almost invariant set (which we will need for the proof of Theorems 6 and 7) is the following:

Lemma 4. Let $\left(m_{i}\right)_{i=0}^{\infty},\left(n_{i}\right)_{i=0}^{\infty}$ be sequences in $\mathbb{N}$ such that $m_{i}<n_{i}<m_{i+1}$ and $m_{i+1}>q m_{i}$ for $i=0,1,2, \ldots$ and some real constant $q>1$. Then the set $A:=\bigcup_{i=0}^{\infty}\left[m_{i}, n_{i}\right]$ is almost invariant.

Proof. For fixed $g \in \mathcal{G}$ let $\phi$ be a positive function on $\mathbb{R}_{+}$, which is decreasing to 0 and satisfies $n \phi(n) \geq|\{i \leq n: g i>n\}|$ and $(n-1) \phi(n-1) \leq n \phi(n), n=1,2, \ldots$.

We can estimate the density function of the set $A \backslash g A$ at $n$ by the number of points crossing the boundary of the set $A \cap[1, n]$ under the action of $g$ :

Since

$$
\begin{aligned}
|[m, n] \backslash g([m, n])| & \leq|\{i \leq m-1: g i>m-1\}|+|\{i \leq n: g i>n\}| \\
& \leq(m-1) \phi(m-1)+n \phi(n),
\end{aligned}
$$

we obtain

$$
\begin{aligned}
n d_{A \backslash g A}(n) & \leq \sum_{m_{i} \leq n}\left(m_{i}-1\right) \phi\left(m_{i}-1\right)+\sum_{n_{i} \leq n} n_{i} \phi\left(n_{i}\right)+(n) \phi(n) \\
& \leq \sum_{m_{i} \leq n} m_{i} \phi\left(m_{i}\right)+\sum_{n_{i} \leq n} n_{i} \phi\left(n_{i}\right)+n \phi(n) \leq 2 \sum_{n_{i} \leq n} n_{i} \phi\left(n_{i}\right)+2 n \phi(n) .
\end{aligned}
$$

For $n \geq m_{0}, 0 \leq i<I(n)$ with $I(n):=\sup \left\{i: m_{i} \leq n\right\}$, we have

$$
q^{i} \leq m_{i}<n_{i}<m_{i+1}=\left(m_{i+1} q^{I(n)-i-1}\right) q^{i+1-I(n)} \leq m_{I(n)} q^{i+1-I(n)}<n q^{i+1-I(n)}
$$


and $n_{i} \phi\left(n_{i}\right)<n q^{i+1-I(n)} \phi\left(q^{i}\right)$ for $i<I(n)$. We thus obtain

$$
d_{A \backslash g A}(n) \leq \frac{2}{n} \sum_{i=0}^{I(n)} n_{i} \phi\left(n_{i}\right)+2 \phi(n)<2 \sum_{i=0}^{I(n)-1} q^{i+1-I(n)} \phi\left(q^{i}\right)+4 \phi(n) .
$$

To see that $\overline{d_{A \backslash g A}}=0$, it remains to show that $S(k):=\sum_{i=0}^{k} q^{i-k} \phi\left(q^{i}\right)$ converges to 0 as $k \rightarrow \infty$ : We have $S(k+1)=S(k) / q+\phi\left(q^{k+1}\right)$, therefore

(5) $\limsup _{k \rightarrow \infty} S(k)=\limsup _{k \rightarrow \infty} S(k+1)=\limsup _{k \rightarrow \infty} S(k) / q+\phi\left(q^{k+1}\right)=\limsup _{k \rightarrow \infty} S(k) / q$.

Since $S(k)$ is bounded by $\phi(1) \sum_{i} q^{-i}$, we obtain $\lim _{k \rightarrow \infty} S(k)=0$.

So the set $A \backslash g A$ has density $0 \forall g \in \mathcal{G}$. As $g A \backslash A=g\left(A \backslash g^{-1} A\right)$ it follows by Lemma 2 that $0=\overline{d_{A \backslash g^{-1} A}}=\overline{d_{g A \backslash A}}$, hence $\overline{d_{A \Delta g A}}=0$ and the set $A$ is almost invariant.

Sets of positive upper density have been studied in combinatorial number theory, since they always contain subsets which exhibit a distinguished behaviour under certain translations. See [10] for an overview. The following Theorem 3, which will be used to describe the closure of the set of almost periodic points in $\beta \mathbb{N}$, shows that sets with positive upper density also contain subsets which have special properties under the action of the Lévy group.

We first prove

Lemma 5. Let $\left(c_{i}\right)$ be a sequence in $\mathbb{N}$ with $c_{i+1}>2 c_{i}$.

(a) For a subset $D$ of $\mathbb{N}$ there exists a subsequence $\left(n_{D, i}\right)$ of $\left(n_{i}\right)$ and an absolutely continuous function $\zeta_{D}$ on the unit interval $I=\{y \in \mathbb{R}: 0 \leq y \leq 1\}$, such that

$$
0 \leq\left((1+x) \zeta_{D}(x)\right)^{\prime} \leq 1 \quad \text { a.e. in } I
$$

and $\lim _{i \rightarrow \infty} d_{D}\left(\left[(1+x) c_{D, i}\right]\right)=\zeta_{D}(x)$ uniformly for $x \in I$.

(b) If the sequence $\left(c_{i}\right)$ satisfies furthermore $\frac{c_{i}}{c_{i+1}} \underset{i \rightarrow \infty}{\longrightarrow} 0$ and if $\zeta$ is an absolutely continuous real-valued function on $I$ with $\zeta(0)=0$ and

$$
0 \leq((1+x) \zeta(x))^{\prime} \leq \frac{1}{m} \quad \text { a.e. in } I
$$

for some $m \in \mathbb{N}$, then there is a subset $D$ of $\bigcup_{i}\left[c_{i}, 2 c_{i}\right]$ with $|D \cap[n, n+m-1]| \leq 1$ $\forall n \in \mathbb{N}$, such that $\lim _{i \rightarrow \infty} d_{D}\left(\left[(1+x) c_{i}\right]\right)=\zeta(x)$ uniformly for $x \in I$.

Proof. We first note that the definition of the density function gives for any $D \subset \mathbb{N}$

$$
d_{D}(n+l)=\frac{n}{n+l} d_{D}(n)+\frac{1}{n+l}|D \cap[n+1, n+l]|,
$$

hence

$$
-\frac{l}{n+l} d_{D}(n) \leq d_{D}(n+l)-d_{D}(n) \leq-\frac{l}{n+l} d_{D}(n)+\frac{l}{n+l} \text { for } n, l \in \mathbb{N} .
$$

We define a sequence of functions $\left(\Psi_{i}\right)$ on $I$ by

$$
\Psi_{i}(x):=\left(\left[x c_{i}\right]+1-c_{i} x\right) d_{D}\left(\left[(1+x) c_{i}\right]\right)+\left(c_{i} x-\left[x c_{i}\right]\right) d_{D}\left(\left[(1+x) c_{i}\right]+1\right) \quad \forall x \in I
$$


i.e. $\Psi_{i}$ is a piecewise linear function with values $\Psi_{i}\left(\frac{l}{c_{i}}\right)=d_{D}\left(c_{i}+l\right)$ at the nodes $\frac{l}{c_{i}}, l=0,1, \ldots, c_{i}$. We now have by (9)

$$
\left|\Psi_{i}\left(\frac{l}{c_{i}}\right)-\Psi_{i}\left(\frac{l+1}{c_{i}}\right)\right|=\left|d_{D}\left(c_{i}+l\right)-d_{D}\left(c_{i}+l+1\right)\right| \leq \frac{1}{c_{i}+l+1}<\frac{1}{c_{i}},
$$

so $\Psi_{i}$ is Lipschitz-continuous with Lipschitz-constant 1 and $\left(\Psi_{i}\right)$ is an equicontinuous family of functions. It now follows from Arzelà-Ascoli's theorem (cf. [6], IV.6.7, I.6.13), that we may pass to a subsequence $\left(\Psi_{D, i}\right)$ of $\left(\Psi_{i}\right)$, such that the functions $\Psi_{D, i}$ converge uniformly on $I$ to some continuous function $\zeta_{D}$. We denote the corresponding subsequence of $\left(c_{i}\right)$ by $\left(c_{D, i}\right)$. Since all functions $\Psi_{i}$ are Lipschitzcontinuous with Lipschitz constant 1 , so is their uniform limit $\zeta_{D}$. Therefore $\zeta_{D}$ is absolutely continuous and the derivative $\zeta_{D}^{\prime}$ exists a.e. in $I$.

Since $d_{D}\left(\left[(1+x) c_{D, i}\right]\right)=\Psi_{D, i}\left(\left[x c_{D, i}\right] / c_{D, i}\right)$ and $\left[x c_{D, i}\right] / c_{D, i} \underset{i \rightarrow \infty}{\longrightarrow} x$ uniformly in $I$, it follows from the uniform convergence of the sequence $\left(\Psi_{D, i}\right)$ to the continuous function $\zeta_{D}$, that $\lim _{i \rightarrow \infty} d_{D}\left(\left[(1+x) c_{D, i}\right]\right)=\zeta_{D}(x)$ uniformly for $x \in I$.

Assume that the sequences $\left(n_{i}\right)$ and $\left(l_{i}\right)$ satisfy $\frac{n_{i}}{c_{i}} \rightarrow 1+x$ and $\frac{l_{i}}{c_{i}} \rightarrow \epsilon$ for some $x \in I$ and $0<\epsilon \leq 1-x$, so that $\lim _{i \rightarrow \infty} d_{D}\left(n_{i}\right)=\zeta_{D}(x)$ and $\lim _{i \rightarrow \infty} d_{D}\left(n_{i}+l_{i}\right)=$ $\zeta_{D}(x+\epsilon)$. Then

$$
\frac{l_{i}}{n_{i}+l_{i}}=\frac{l_{i} / c_{D, i}}{n_{i} / c_{D, i}+l_{i} / c_{D, i}} \underset{i \rightarrow \infty}{\longrightarrow} \frac{\epsilon}{1+x+\epsilon}
$$

and we obtain with (9)

Hence

$$
-\frac{\epsilon}{1+x+\epsilon} \zeta_{D}(x) \leq \zeta_{D}(x+\epsilon)-\zeta_{D}(x) \leq-\frac{\epsilon}{1+x+\epsilon} \zeta_{D}(x)+\frac{\epsilon}{1+x+\epsilon} .
$$

$$
(1+x) \zeta_{D}(x) \leq(1+x+\epsilon) \zeta_{D}(x+\epsilon) \leq(1+x) \zeta_{D}(x)+\epsilon
$$

and

$$
0 \leq \frac{(1+x+\epsilon) \zeta_{D}(x+\epsilon)-(1+x) \zeta_{D}(x)}{\epsilon} \leq 1
$$

Therefore

$$
0 \leq\left((1+x) \zeta_{D}(x)\right)^{\prime} \leq 1
$$

if this derivative exists in $x$. As we observed above $\zeta_{D}$ is differentiable a.e. in $I$, so (6) follows.

To prove part (b) of the lemma let $\varrho$ for $n \in\left[c_{i}, 2 c_{i}\right]$ be given by

$$
\varrho(n)=\zeta\left(\frac{n-c_{i}}{c_{i}}\right) \text {. }
$$

We now define a subset $D$ of $\bigcup_{i}\left[c_{i}, 2 c_{i}\right]$ in the obvious way inductively as follows: $c_{i} \notin D$ and for $c_{i}<n \leq 2 c_{i}$

$$
n \in D \Leftrightarrow\left(d_{D}(n-1)<\varrho(n-1),[n-m+1, n-1] \cap D=\emptyset\right) .
$$

We claim that $\lim _{n \rightarrow \infty} d_{D}(n)-\varrho(n)=0$ as $n \rightarrow \infty$ in $\bigcup_{i}\left[c_{i}, 2 c_{i}\right]$. We first note that since $\zeta$ is absolutely continuous, so is the function $t \rightarrow(1+t) \zeta(t)$ on $I$, and we have (cf. [11], 18.16)

$$
(1+x+y) \zeta(x+y)-(1+x) \zeta(x)=\int_{x}^{x+y}((1+t) \zeta(t))^{\prime} d t
$$


for $x, y, x+y \in I$, so (7) yields

$$
\frac{1+x}{1+x+y} \zeta(x) \leq \zeta(x+y) \leq \frac{1+x}{1+x+y} \zeta(x)+\frac{1}{m} \frac{y}{1+x+y} .
$$

With (10) we obtain by setting $y=k / c_{i}$ and $x=\left(n-c_{i}\right) / c_{i}$

$$
\frac{n}{n+k} \varrho(n) \leq \varrho(n+k) \leq \frac{n}{n+k} \varrho(n)+\frac{1}{m} \frac{k}{n+k}
$$

for $c_{i} \leq n \leq 2 c_{i}, \quad 0 \leq k \leq 2 c_{i}-n$.

For $d_{D}(n) \geq \varrho(n)$ our definition of the set $D$ implies $n+1 \notin D$, so $d_{D}(n+1)=$ $\frac{n}{n+1} d_{D}(n)$ and we obtain with (11)

$$
\begin{aligned}
d_{D}(n+1)-\varrho(n+1) & \leq \frac{n}{n+1} d_{D}(n)-\frac{n}{n+1} \varrho(n) \\
& =\frac{n}{n+1}\left(d_{D}(n)-\varrho(n)\right) \leq d_{D}(n)-\varrho(n) .
\end{aligned}
$$

Thus if we have $d_{D}\left(n_{0}\right) \leq \varrho\left(n_{0}\right)$ for some $n_{0}, c_{i} \leq n_{0}<n \leq 2 c_{i}$ and $d_{D}(l) \geq \varrho(l)$ for $l=n_{0}+1, \ldots, n$ we obtain from (9), (11) and (12):

$$
\begin{aligned}
d_{D}(n)-\varrho(n) & \leq d_{D}\left(n_{0}+1\right)-\varrho\left(n_{0}+1\right) \\
& \leq \frac{n_{0}}{n_{0}+1} d_{D}\left(n_{0}\right)+\frac{1}{n_{0}+1}-\frac{n_{0}}{n_{0}+1} \varrho\left(n_{0}\right) \leq \frac{1}{n_{0}+1} \leq \frac{1}{c_{i}} .
\end{aligned}
$$

Otherwise, if no such $n_{0}$ exists, i.e. if $d_{D} \geq \varrho$ in $\left[c_{i}, n\right]$, then $D \cap\left[c_{i}, n\right]$ is empty and $d_{D}$ is decreasing in $\left[c_{i}, n\right]$. We then have $d_{D}(n)-\varrho(n) \leq d_{D}\left(c_{i}\right)$.

Together with (13) we obtain $\forall n \in\left[c_{i}, 2 c_{i}\right]$

$$
d_{D}(n)-\varrho(n) \leq \max \left(d_{D}\left(c_{i}\right), 1 / c_{i}\right) .
$$

As $c_{i} / c_{i+1} \rightarrow 0$ and $D$ is supported in $\bigcup_{i}\left[c_{i}, 2 c_{i}\right]$ we have $d_{D}\left(c_{i}\right) \rightarrow 0$ and obtain

$$
\varlimsup_{n \rightarrow \infty} d_{D}(n)-\varrho(n) \leq 0
$$

as $n$ tends to $\infty$ in $\bigcup_{i}\left[c_{i}, 2 c_{i}\right]$.

To obtain a lower bound for $d_{D}-\varrho$ assume that $d_{D}(n)-\varrho(n)<0$ for some $n \in\left[c_{i}, 2 c_{i}\right]$. As $\varrho\left(c_{i}\right)=\zeta(0)=0$ and $d_{D}\left(c_{i}\right) \geq 0$ there is an $n_{0}, c_{i} \leq n_{0}<2 c_{i}$ with $d_{D}\left(n_{0}\right)-\varrho\left(n_{0}\right) \geq 0$, such that $d_{D}(l)<\varrho(l)$ holds for $n_{0}<l \leq n$. Our definition of the set $D$ guarantees that for $n_{0}<l \leq n-m$ there is exactly one element of $[l+1, l+m]$ in $D$, so $d_{D}(l+m)=\frac{l}{l+m} d_{D}(l)+\frac{1}{l+m}$ by (8), and we obtain with (11)

$$
\begin{aligned}
d_{D}(l+m)-\varrho(l+m) & \geq \frac{l}{l+m} d_{D}(l)+\frac{1}{l+m}-\frac{l}{l+m} \varrho(l)-\frac{1}{m} \frac{m}{l+m} \\
& =\frac{l}{l+m}\left(d_{D}(l)-\varrho(l)\right) \geq d_{D}(l)-\varrho(l)
\end{aligned}
$$

for $l \in\left[n_{0}+1, n-m\right]$.

For $n_{0}<l \leq n_{0}+m$ we have by (8) and (11)

$$
d_{D}(l)-\varrho(l) \geq \frac{n_{0}}{l} d_{D}\left(n_{0}\right)-\frac{n_{0}}{l} \varrho\left(n_{0}\right)-\frac{1}{m} \frac{l-n_{0}}{l} \geq-\frac{1}{m} \frac{l-n_{0}}{l} \geq-\frac{1}{l} \geq-\frac{1}{c_{i}} .
$$

Applying the estimate (15) repeatedly for $l=n-m, n-2 m, \ldots, n-j m$, where $n_{0}<n-j m \leq n_{0}+m$ this yields $d_{D}(n)-\varrho(n) \geq d_{D}(n-j m)-\varrho(n-j m) \geq-\frac{1}{c_{i}}$ for $n \in\left[c_{i}, 2 c_{i}\right]$ and we obtain

$$
\varlimsup_{n \rightarrow \infty} d_{D}(n)-\varrho(n) \geq 0
$$

as $n$ tends to $\infty$ in $\bigcup_{i}\left[c_{i}, 2 c_{i}\right]$. 
It follows from (14) and (16) that $d_{D}(n)-\varrho(n)$ converges to 0 in $\bigcup_{i}\left[c_{i}, 2 c_{i}\right]$.

As $\varrho\left(\left[(1+x) c_{i}\right]\right)=\zeta\left(\frac{\left[x c_{i}\right]}{c_{i}}\right)$ and $\frac{\left[x c_{i}\right]}{c_{i}} \underset{i \rightarrow \infty}{\longrightarrow} x$ uniformly on $I$ the uniform convergence of $d_{D}\left(\left[(1+x) c_{i}\right]\right)$ to $\zeta(x)$ as $i \rightarrow \infty$ follows from the uniform continuity of $\zeta$.

Corollary 1. Let $A_{1}, A_{2}$ be disjoint subsets of $\mathbb{N}$ and let $\left(c_{i}\right)$ be a sequence in $\mathbb{N}$ with $c_{i+1}>2 c_{i}$, such that

$$
d_{A_{1}}\left(\left[(1+x) c_{i}\right]\right) \underset{i \rightarrow \infty}{\longrightarrow} \zeta_{A_{1}}(x) \quad \text { and } \quad d_{A_{2}}\left(\left[(1+x) c_{i}\right]\right) \underset{i \rightarrow \infty}{\longrightarrow} \zeta_{A_{2}}(x) \text { a.e. in } I .
$$

Then

$$
d_{A_{1} \cup A_{2}}\left(\left[(1+x) c_{i}\right]\right) \underset{i \rightarrow \infty}{\longrightarrow} \zeta_{A_{1} \cup A_{2}}(x) \text { with } \zeta_{A_{1} \cup A_{2}}=\zeta_{A_{1}}+\zeta_{A_{2}} \text { a.e. }
$$

Furthermore the functions $\zeta_{A_{1}}, \zeta_{A_{2}}$ and $\zeta_{A_{1} \cup A_{2}}$ are absolutely continuous on I and $\left((1+x) \zeta_{A_{1}}(x)\right)^{\prime}=0$ a.e. in some Borel subset $E$ of $I$ if $\left((1+x) \zeta_{A_{1} \cup A_{2}}(x)\right)^{\prime}=0$, or if $\left((1+x) \zeta_{A_{2}}(x)\right)^{\prime}=1$ a.e. in $E$.

Proof. Since for disjoint subsets $A_{1}, A_{2}$ of $\mathbb{N}$ we have $d_{A_{1} \cup A_{2}}=d_{A_{1}}+d_{A_{2}}$, the convergence to the function $\zeta_{A_{1}}+\zeta_{A_{2}}$ is obvious.

The second claim now follows from

$$
\left((1+x) \zeta_{A_{1} \cup A_{2}}(x)\right)^{\prime}=\left((1+x) \zeta_{A_{1}}(x)\right)^{\prime}+\left((1+x) \zeta_{A_{2}}(x)\right)^{\prime}
$$

and Lemma 5 (a), since the functions $\zeta_{A_{1}}, \zeta_{A_{2}}, \zeta_{A_{1} \cup A_{2}}$ all are absolutely continuous with their derivatives taking values between 0 and 1 only a.e. in $I$.

Theorem 3. Let $A$ be a subset of $\mathbb{N}$ with positive upper density and set $m=$ $\left[2 / \overline{d_{A}}\right]+1$, then there are $g_{1}, \ldots, g_{m} \in \mathcal{G}$ such that $\bigcup_{i=1}^{m} g_{i} A$ contains an almost invariant set $B$ with $\overline{d_{B}}>0$.

Proof. Let $\left(c_{i}\right)$ be a sequence in $\mathbb{N}$ with $\lim _{i \rightarrow \infty} d_{A}\left(2 c_{i}\right)=\overline{d_{A}}, c_{i+1}>2 c_{i}$ and $c_{i} / c_{i+1} \underset{i \rightarrow \infty}{\longrightarrow} 0$. By Lemma 5 (a) there is a subsequence $\left(c_{A, i}\right)$ of $\left(c_{i}\right)$ and an absolutely continuous function $\zeta_{A}$ satisfying (6), such that

$$
\lim _{i \rightarrow \infty} d_{A}\left(\left[(1+x) c_{A, i}\right]\right)=\zeta_{A}(x) \text { uniformly for } x \in I .
$$

Then $\zeta_{A}(x) \leq \overline{d_{A}} \forall x \in I$ and $\zeta_{A}(1)=\overline{d_{A}}$. Since $\zeta_{A}$ is absolutely continuous, there exists a Borel subset $E$ of $I$ with positive Lebesgue measure, such that $\zeta_{A}^{\prime}(x) \geq 0$ and $\zeta_{A}(x) \geq \overline{d_{A}} / 2>1 / m$ for $x \in E$. (In fact by the continuity of $\zeta_{A}$ there is an $\alpha<1$ with $\zeta_{A}(x) \geq \overline{d_{A}} / 2=\zeta_{A}(1) / 2>0$ for $\alpha \leq x \leq 1$. If $\zeta_{A}^{\prime}$ were negative a.e. in $\{y: \alpha \leq y \leq 1\}$, then by [11], 18.16 we would obtain $\zeta_{A}(\alpha)>\zeta_{A}(1)=\overline{d_{A}}$ contradicting (17).) It follows with (6) that

$$
\left((1+x) \zeta_{A}(x)\right)^{\prime}>\frac{1}{m} \text { for } x \in E, \quad 0 \leq\left((1+x) \zeta_{A}(x)\right)^{\prime} \leq 1 \text { a.e. in } I .
$$

We define the absolutely continuous function

$$
\zeta(x):=\frac{1}{m} \frac{1}{1+x} \int_{0}^{x} \chi_{E}(t) d t, \quad x \in I, \text { so }((1+x) \zeta(x))^{\prime}=\frac{1}{m} \chi_{E}(x) \text { a.e. in } I
$$


by [11], 18.17 and inequality (7) is satisfied. Applying Lemma 5 (b) to the sequence $\left(c_{A, i}\right)$ we find a subset $B_{0}$ of $\bigcup_{i}\left[c_{A, i}, 2 c_{A, i}\right]$ with

$$
\lim _{i \rightarrow \infty} d_{B_{0}}\left(\left[(1+x) c_{A, i}\right]\right)=\zeta(x) \quad \text { uniformly in } I .
$$

We now define the set $B$ by

$$
B=\left\{n \in \bigcup_{i}\left[c_{A, i}, 2 c_{A, i}\right]:[n-m+1, n] \cap B_{0} \neq \emptyset\right\} .
$$

So $B$ is essentially (up to the negligible boundaries of the intervals $\left[c_{A, i}, 2 c_{A, i}\right]$ ) the union of $m$ pairwise disjoint right translates of $B_{0}$. We therefore have $d_{B}(n)-$ $m d_{B_{0}}(n) \underset{n \rightarrow \infty}{\longrightarrow} 0$ and with $(20)$

$$
\lim _{i \rightarrow \infty} d_{B}\left(\left[(1+x) c_{A, i}\right]\right)=: \zeta_{B}(x)=m \zeta(x) \quad \text { uniformly in } I .
$$

We have to prove that $B$ is an almost invariant subset of $\mathbb{N}$. We first show that for $g \in \mathcal{G}$ and $C_{g}:=g B \backslash B$ we have $\overline{d_{C_{g}}}=0$ :

Assume that

$$
\lim _{i \rightarrow \infty} \sup _{n \in\left[\tilde{c}_{i}, 2 \tilde{c}_{i}\right]} d_{C_{g}}(n)=: \Gamma>0
$$

for some subsequence $\left(\tilde{c}_{i}\right)$ of $\left(c_{A, i}\right)$. We then apply Lemma 5 (a) to the sequence $\left(\tilde{c}_{i}\right)$ and the set $C_{g}$ to obtain a subsequence $\left(c_{C_{g}, i}\right)$ of $\left(\tilde{c}_{i}\right)$ and an absolutely continuous function $\zeta_{C_{g}}$ for which

(22) $\lim _{i \rightarrow \infty} d_{C_{g}}\left(\left[(1+x) c_{C_{g}, i}\right]\right)=\zeta_{C_{g}}(x)$ uniformly in $I$, and $\max _{x \in I} \zeta_{C_{g}}(x)=\Gamma>0$.

Since $B \cap C_{g}=\emptyset$ it follows from Corollary 1 that $\left((1+x) \zeta_{C_{g}}(x)\right)^{\prime}=0$ a.e. on $E$, since $\left((1+x) \zeta_{B}(x)\right)^{\prime}=1$ a.e. on $E$ by $(19),(21)$.

From Lemma 2 and (21) it follows that

$$
\lim _{i \rightarrow \infty} d_{g B}\left(\left[(1+x) c_{A, i}\right]\right)=\zeta_{B}(x) \quad \text { uniformly in } I .
$$

Since $g B$ is the disjoint union of the sets $C_{g}$ and $g B \cap B$ it follows with (22) and (23) that

$$
\lim _{i \rightarrow \infty} d_{g B \cap B}\left(\left[(1+x) c_{A, i}\right]\right)=\zeta_{B}(x)-\zeta_{C_{g}}(x) \text { uniformly in } I .
$$

Applying Corollary 1 again to the disjoint sets $C_{g}$ and $g B \cap B$ we see that $\left((1+x) \zeta_{C_{g}}(x)\right)^{\prime}=0$ a.e. on $I \backslash E$, since $\left((1+x) \zeta_{B}(x)\right)^{\prime}=0$ a.e. on $I \backslash E$ by (19), (21) and therefore $\left((1+x)\left(\zeta_{B}-\zeta_{C_{g}}\right)(x)\right)^{\prime}=0$ a.e. in $I \backslash E$.

Hence $\left((1+x) \zeta_{C_{g}}\right)^{\prime}=0$ a.e. in $I$.

Since $\zeta_{C_{g}}$ is absolutely continuous by Lemma 5 and $\zeta_{C_{g}}(0)=0$ it follows (cf. [11], 18.16) that $(1+x) \zeta_{C_{g}}(x)=\int_{0}^{x}\left((1+t) \zeta_{C_{g}}(t)\right)^{\prime} d t=0 \quad \forall x \in I$, which is a contradiction to $\Gamma>0$ in (22).

Hence for all $g \in \mathcal{G}$ we have $\overline{d_{C_{g}}}=0$. Since $B \backslash g B=g\left(g^{-1} B \backslash B\right)=g C_{g^{-1}}$ it follows with Lemma 2 that $\overline{d_{B \backslash g B}}=0$. With $\overline{d_{C_{g}}}=0$ this yields $\overline{d_{B \triangle g B}}=0$, so $B$ is almost invariant.

It remains to show that $B \subset \bigcup_{j=0}^{m-1} g_{j} A$ for some $g_{0}, g_{1}, \ldots, g_{m-1} \in \mathcal{G}$.

We divide $B$ into $m$ mutually disjoint subsets $B_{0}, B_{1}, \ldots, B_{m-1}$ by translating $B_{0}$ : we set $B_{j}=\left\{n \in B: n-j \in B_{0}\right\} \cap\left(\bigcup_{i}\left[c_{A, i}, 2 c_{A, i}\right]\right)$.

It easily follows that $d_{B_{j}}(n)-d_{B_{0}}(n) \underset{n \rightarrow \infty}{\longrightarrow} 0$, so by $(20)$

$$
\lim _{i \rightarrow \infty} d_{B_{j}}\left(\left[(1+x) c_{A, i}\right]\right)=\zeta(x) \text { uniformly for } x \in I, \quad j=0, \ldots, m-1 .
$$


We now define inductively injective maps $\rho_{j}$ from $B_{j} \backslash A$ to $A \backslash B_{j}$ as follows: For $k \in B_{j} \backslash A$ set

$$
\rho_{j}(k)=\min \left\{l \in A \backslash B_{j}: l>k, l \notin \rho_{j}\left(\left\{n \in B_{j} \backslash A: n<k\right\}\right)\right\} .
$$

Since $((1+x) \zeta(x))^{\prime}=\frac{1}{m} \chi_{E}$ a.e. by (19) and $\frac{1}{m} \chi_{E} \leq\left((1+x) \zeta_{A}(x)\right)^{\prime}$ a.e. on $I$ with the inequality being strict on $E$ by (18), it follows from the absolute continuity of these functions and $\zeta_{A}(0) \geq \zeta(0)=0$ that $\zeta_{A}(1)>\zeta(1)$ (cf. [11], 18.16). Hence $\lim _{i \rightarrow \infty} d_{A}\left(2 c_{A, i}\right)>\lim _{i \rightarrow \infty} d_{B_{j}}\left(2 c_{A, i}\right)$, so $A \backslash B_{j}$ is infinite and this definition makes sense.

We define permutations $g_{j}, j=0, \ldots, m-1$ as the product of two-cycles:

$$
g_{j}=\prod_{n \in B_{j} \backslash A}\left(n, \rho_{j}(n)\right), \quad \text { so } g_{j}(n)= \begin{cases}\rho_{j}(n), & n \in B_{j} \backslash A, \\ \rho_{j}^{-1}(n), & n \in \rho_{j}\left(B_{j} \backslash A\right), \\ n, & \text { otherwise, }\end{cases}
$$

and claim that $g_{j} \in \mathcal{G}$. We have to show that

$$
\phi_{j}(n):=\frac{1}{n}\left|\left\{l: l \leq n, g_{j}(l)>n\right\}\right|=\frac{1}{n}\left|\left\{l \in B_{j} \backslash A: l \leq n, \rho_{j}(l)>n\right\}\right|
$$

tends to 0 for $j=0, \ldots, m-1$. We first note that by our definition of $\rho_{j}$ we have

$$
(n+1) \phi_{j}(n+1)-n \phi_{j}(n)=\left\{\begin{array}{lll}
1, & n+1 \in B_{j} \backslash A, & \\
-1, & n+1 \in A \backslash B_{j}, & \phi_{j}(n)>0, \\
0, & n+1 \in A \backslash B_{j}, & \phi_{j}(n)=0, \\
0, & n+1 \notin A \Delta B_{j}, &
\end{array}\right.
$$

and it follows that if $\phi_{j}>0$ in $[k+1, l-1]$ for some $l, k \in \mathbb{N}, k<l-1$, then $\phi_{j}(k)>0$ or $k+1 \in B_{j} \backslash A$ and (25) yields

$$
\begin{aligned}
l \phi_{j}(l)-k \phi_{j}(k) & =\left|B_{j} \cap[k+1, l]\right|-|A \cap[k+1, l]| \\
& =l\left(d_{B_{j}}(l)-d_{A}(l)\right)-k\left(d_{B_{j}}(k)-d_{A}(k)\right) .
\end{aligned}
$$

For $l \in\left[c_{A, i}, 2 c_{A, i}\right]$ we set $k:=\max \left(\left\{n \in \mathbb{N}: c_{A, i} \leq n \leq l, \phi_{j}(n)=0\right\} \cup\left\{c_{A, i}\right\}\right)$, so we have $k \phi(k) \leq c_{A, i} \phi\left(c_{A, i}\right)$ and obtain

$$
\begin{aligned}
\phi_{j}(l) & \leq \frac{l}{c_{A, i}} \phi_{j}(l)=\frac{1}{c_{A, i}}\left(k \phi_{j}(k)+l\left(d_{B_{j}}(l)-d_{A}(l)\right)-k\left(d_{B_{j}}(k)-d_{A}(k)\right)\right) \\
& \leq \phi_{j}\left(c_{A, i}\right)+\frac{l}{c_{A, i}}\left(d_{B_{j}}(l)-d_{A}(l)\right)-\frac{k}{c_{A, i}}\left(d_{B_{j}}(k)-d_{A}(k)\right) .
\end{aligned}
$$

By $(24)$ and (17) there is a sequence $\left(\epsilon_{i}\right)$ converging to 0 , such that

$$
\left|\zeta\left(\frac{n}{c_{A, i}}-1\right)-d_{B_{j}}(n)\right|<\epsilon_{i}, \quad\left|\zeta_{A}\left(\frac{n}{c_{A, i}}-1\right)-d_{A}(n)\right|<\epsilon_{i}
$$

for all $n \in\left[c_{A, i}, 2 c_{A, i}\right], i \in \mathbb{N}$ and we obtain from (26)

$$
\begin{aligned}
\phi_{j}(l) \leq & \phi_{j}\left(c_{A, i}\right)+8 \epsilon_{i}+\frac{l}{c_{A, i}}\left(\zeta\left(\frac{l}{c_{A, i}}-1\right)-\zeta_{A}\left(\frac{l}{c_{A, i}}-1\right)\right) \\
& -\frac{k}{c_{A, i}}\left(\zeta\left(\frac{k}{c_{A, i}}-1\right)-\zeta_{A}\left(\frac{k}{c_{A, i}}-1\right)\right) .
\end{aligned}
$$


Now by the absolute continuity of $\zeta$ and $\zeta_{A}$ and (18), (19) we get (cf. [11], 18.17)

$$
\begin{gathered}
\frac{l}{c_{A, i}}\left(\zeta\left(\frac{l}{c_{A, i}}-1\right)-\zeta_{A}\left(\frac{l}{c_{A, i}}-1\right)\right)-\frac{k}{c_{A, i}}\left(\zeta\left(\frac{k}{c_{A, i}}-1\right)-\zeta_{A}\left(\frac{k}{c_{A, i}}-1\right)\right) \\
=\int_{\frac{k}{c_{A, i}}-1}^{\frac{l}{c_{A, i}}-1}((1+s) \zeta(s))^{\prime}-\left((1+s) \zeta_{A}(s)\right)^{\prime} d s \\
=\int_{\frac{k}{c_{A, i}}-1}^{\frac{l}{c_{A, i}}-1} \frac{1}{m} \chi_{E}(s)-\left((1+s) \zeta_{A}(s)\right)^{\prime} d s<0,
\end{gathered}
$$

so (27) yields

$$
\phi_{j}(l) \leq \phi_{j}\left(c_{A, i}\right)+8 \epsilon_{i} \quad \forall l \in\left[c_{A, i}, 2 c_{A, i}\right] .
$$

Since $B_{j} \subset \bigcup_{i}\left[c_{A, i}, 2 c_{A, i}\right]$, it follows that $\phi_{j}$ is decreasing in $\left[2 c_{A, i}, c_{A, i+1}[\right.$. Since $c_{A, i} / c_{A, i+1} \rightarrow 0$ it follows that $\phi_{j}\left(c_{A, i}\right) \rightarrow 0$ as $i \rightarrow \infty$, and with (28) it follows that $\phi_{j}(n) \rightarrow 0$ as $n \rightarrow \infty$. So we have shown that $g_{j} \in \mathcal{G}$ for $j=0, \ldots, m-1$.

We define

$$
S:=\{p \in \beta \mathbb{N}: \text { the ultrafilter } p \text { contains no set of density } 0\} .
$$

This subset of $\beta \mathbb{N}$ has been used to investigate topological and algebraic properties of $\beta \mathbb{N}$. It can be shown that no point in $S$ is the limit point of a countable number of P-points of $\mathbb{N}^{*}$ (points $p$ with the property that the countable intersection of neighborhoods of $p$ is a neighborhood of $p$ ) cf. [20], 7.14. It is shown in [5], 7, that both $S$ and $\mathbb{N}^{*} \backslash S$ are right ideals of the left topological semigroups $(\beta \mathbb{N},+)$ and $(\beta \mathbb{N}, \cdot)$. It follows that the centers of both $(\beta \mathbb{N},+)$ and $(\beta \mathbb{N}, \cdot)$ are $\mathbb{N}$.

We need some properties of this set $S$, some of which are well known; cf. [5], $7.2,[20], 7.15$.

Lemma 6. $S$ is a closed nonempty $\mathcal{G}$-invariant subset of $\mathbb{N}^{*}$ with empty interior. If $A \subset \mathbb{N}$, then $\bar{A} \cap S \neq \emptyset$ iff $\overline{d_{A}}>0$ and $A$ is almost invariant iff the set $\bar{A} \cap S$ is $\mathcal{G}$-invariant.

Proof. Since the finite intersection of subsets of $\mathbb{N}$ with density 1 has density 1 , the collection $\mathfrak{F}$ of these sets is a filter. Any ultrafilter containing $\mathfrak{F}$ is in $S$ since the complement in $\mathbb{N}$ of a set of density 0 has density 1 , so no set of density 0 can be contained in an ultrafilter containing $\mathfrak{F}$.

If $p \notin S$ there exists $B \subset \mathbb{N}$ with $\overline{d_{B}}=0$ and $p \in \bar{B}$. Since $\bar{B}$ is open and $\bar{B} \cap S=\emptyset$ it follows that $S$ is closed. Clearly $S$ contains no points of $\mathbb{N}$, i.e. $S \subset \mathbb{N}^{*}$. Since any subset of $\mathbb{N}$ contains a subset of density 0 no clopen set $\bar{A}, A \subseteq \mathbb{N}$, is contained in $S$, so $S$ has empty interior.

If $\overline{d_{A}}>0$ and $B$ has density 1 we have $\overline{d_{A \cap B}}=\overline{d_{A}}$ and $A \cap B \neq \emptyset$, hence $\mathfrak{F} \cap A$ is a filter base and $\bar{A} \cap S$ is nonempty since any filter contains an ultrafilter by Zorn's lemma. In particular $S$ is nonempty.

If $\bar{A} \cap S \neq \emptyset$, then $\exists p \in \bar{A} \cap S$, hence $A \in p$, so $\overline{d_{A}}>0$ since $p \in S$.

$S$ is $\mathcal{G}$-invariant since by Lemma $2 \overline{d_{A}}>0$ iff $\overline{d_{g A}}>0$, so $p \in S \Leftrightarrow g p \in S$ for $g \in \mathcal{G}$.

It follows that $A$ is almost invariant iff $\overline{A \Delta g A} \cap S=\emptyset \quad \forall g \in \mathcal{G}$. 
We next observe that for $A, B \subset \mathbb{N}$ we have

$$
\begin{aligned}
\overline{A \backslash B} & =\{p \in \beta \mathbb{N}: A \backslash B \in p\} \\
& =\{p \in \beta \mathbb{N}: A \in p \wedge B \notin p\}=\bar{A} \backslash \bar{B} .
\end{aligned}
$$

Since $g \bar{A}=\overline{g A}$ we have $\overline{A \Delta g A}=\overline{A \backslash g A} \cup \overline{g A \backslash A}=(\bar{A} \backslash g \bar{A}) \cup(g \bar{A} \backslash \bar{A})$, so

$$
\begin{aligned}
\overline{A \Delta g A} \cap S=\emptyset & \Leftrightarrow((\bar{A} \backslash g \bar{A}) \cap S) \cup((g \bar{A} \backslash \bar{A}) \cap S)=\emptyset \\
& \Leftrightarrow((\bar{A} \cap S) \backslash(g \bar{A} \cap S)) \cup((g \bar{A} \cap S) \backslash(\bar{A} \cap S))=\emptyset \\
& \Leftrightarrow \bar{A} \cap S=g \bar{A} \cap S .
\end{aligned}
$$

Since $S$ is $\mathcal{G}$-invariant $g \bar{A} \cap S=g(\bar{A} \cap S)$, so $A$ is almost invariant iff $\bar{A} \cap S=g(\bar{A} \cap S)$ $\forall g \in \mathcal{G}$, i.e. iff $\bar{A} \cap S$ is $\mathcal{G}$-invariant.

Among the properties we will investigate is almost periodicity. We recall the pertinent definitions:

A subset $A$ of a topological group $G$ is syndetic if there is a compact subset $K$ of $G$ such that $G=K A$. A point $p$ in the phase space $X$ of the dynamical system $(G, X)$ is almost periodic if for each neighborhood $U$ of $p$ there exists a syndetic subset $A$ such that $g p \in U \forall g \in A$. Our group $\mathcal{G}$ is discrete, so $K$ will be finite. (For abelian group actions these points are called uniformly recurrent in [8].)

In the dynamical system $(G, X)$ the point $p \in X$ has minimal orbit closure if $\overline{G p}$ does not contain nontrivial closed invariant subsets.

For our dynamical system $(\mathcal{G}, \beta \mathbb{N})$ these properties coincide since we have

Lemma 7. For a dynamical system $(G, X), X$ compact, a point $p \in X$ is almost periodic iff $p$ has minimal orbit closure.

For the proof see [19], IV 1.2 or [9], 4.05, 4.10.

We are now ready to prove the main theorem of this section. We recall that the support $\operatorname{supp}(\mu)$ of a (signed) measure $\mu$ is the smallest closed set with the property that any continuous function supported in the complement of this set is in the annihilator of $\mu$. The support set of $\mathcal{G}$-invariant measures is the smallest closed set containing the support of any $\mathcal{G}$-invariant measure.

Theorem 4. For $x \in \mathbb{N}^{*}$ the following are equivalent:

(i) $x \in S$;

(ii) the $\mathcal{G}_{\delta}$-orbit $\mathcal{G}_{\delta} x$ is not dense in $\mathbb{N}^{*}$;

(iii) the $\mathcal{G}$-orbit $\mathcal{G} x$ is not dense in $\mathbb{N}^{*}$;

(iv) $x$ is in the support set of $\mathcal{G}$-invariant measures;

(v) $T f(x)=T f(g x) \quad \forall f \in C(\beta \mathbb{N}), \forall g \in \mathcal{G}$;

(vi) $f(x)=f(g x)$ for all almost invariant functions $f \in C(\beta \mathbb{N})$ and $g \in \mathcal{G}$;

(vii) $x$ is in the closure of the set of points which are almost periodic with respect to $\mathcal{G}$.

Proof. We will first show (i) $\Rightarrow$ (ii) $\Rightarrow$ (iii) $\Rightarrow$ (i). Using the equivalence of (i) and (iii) we then show (i) $\Rightarrow$ (iv) $\Rightarrow$ (iii), (i) $\Rightarrow$ (v) $\Rightarrow$ (iii), (i) $\Rightarrow$ (vi) $\Rightarrow$ (iii) and (i) $\Rightarrow$ (vii) $\Rightarrow$ (iii), where the implication (ii) $\Rightarrow$ (iii) is obvious.

(i) $\Rightarrow$ (ii): Assume $y$ has dense orbit under the action of $\mathcal{G}_{\delta}$. Taking any infinite set $B \subset \mathbb{N}$ with density 0 , then $\exists g \in \mathcal{G}_{\delta}$ such that $g y \in \bar{B}$ since $\bar{B}$ is open. Then 
$y \in g^{-1} \bar{B}=\overline{g^{-1} B}$. This shows that the ultrafilter $y$ contains a set of density 0 since $\mathcal{G}_{\delta}$ is density preserving hence $y \notin S$.

(iii) $\Rightarrow$ (i): For $y \in \mathbb{N}^{*} \backslash S$ we can find an infinite subset $A$ of $\mathbb{N}$ of density 0 with $y \in \bar{A}$. Let $B$ be an arbitrary infinite subset of $\mathbb{N}$ and $B_{0} \subset B$ an infinite subset of $B$ of density 0 . By Lemma $3 \exists g \in \mathcal{G}$ such that $g A=B_{0}$. Therefore $g y \in \overline{B_{0}} \subset \bar{B}$. Since the collection of clopen sets $\bar{B}, B$ an infinite subset of $\mathbb{N}$, is a base for the topology of $\mathbb{N}^{*}$, it follows that the $\mathcal{G}$-orbit of $y$ is dense in $\mathbb{N}^{*}$.

(i) $\Rightarrow$ (iv): Assume that $A \in x$ for some $A \subset \mathbb{N}$. Since $x \in S$ the function $T \chi_{\bar{A}}$ (which is just the continuous extension of $d_{A}$ to $\beta \mathbb{N}$ ) does not vanish on $\mathbb{N}^{*}$, so there is a $q \in \mathbb{N}^{*}$, such that $T \chi_{\bar{A}}(q)=\left\langle T^{*} \delta_{q}, \chi_{\bar{A}}\right\rangle \neq 0$. By Theorem 2 the measure $T^{*} \delta_{q}$ is $\mathcal{G}$-invariant and has support intersecting $\bar{A}$. As the collection of sets $\bar{A}$, $x \in \bar{A}, A \subset \mathbb{N}$, is a neighborhood base for $x$, it follows that $x$ is in the support set of $\mathcal{G}$-invariant measures.

(iv) $\Rightarrow$ (iii): Since there exists an uncountable number of pairwise almost disjoint infinite subsets of $\mathbb{N}$ (sets with pairwise finite intersection) cf. [20], 3.21, there exists an uncountable number of pairwise disjoint open subsets of $\mathbb{N}^{*}$. Therefore no measure on $\mathbb{N}^{*}$ can have $\mathbb{N}^{*}$ as its support. But if $\mu$ is $\mathcal{G}$-invariant, then the support of $\mu$ is $\mathcal{G}$-invariant. Therefore $\mathcal{G} y$ cannot be dense in $\mathbb{N}^{*}$ for any $y$ in the support of $\mu$. So the support of any $\mathcal{G}$-invariant measure is contained in $S$ by the equivalence of (i) and (iii). Since $S$ is closed by Lemma 6 , it follows that the support set of $\mathcal{G}$-invariant measures is contained in $S$.

(i) $\Rightarrow(\mathrm{v})$ : Let $g \in \mathcal{G}, f \in C(\beta \mathbb{N})$ with $T f(y)=1, T f(g y)=0$ for some $y \in \mathbb{N}^{*}$. Let $B_{1} \subset \mathbb{N}$ be in the ultrafilter $y$, such that $T f(n)>\frac{2}{3}$ for $n \in B_{1}$, and $B_{0} \subset \mathbb{N}$ in the ultrafilter $g y$, such that $T f(n)<\frac{1}{3}$ for $n \in B_{0}$. Now $y \in \overline{B_{1}}$ and $g y \in \overline{B_{0}}$ implies $y \in \bar{A}$ with $A:=B_{1} \cap g^{-1} B_{0}$. So $A \in y$ and $g A \in g y$. We want to show that $A$ has density 0, i.e. $y \notin S$.

Since the sets $A$ and $g A$ are disjoint, there are numbers

$$
M_{1} \leq N_{1} \leq \ldots<M_{i} \leq N_{i}<M_{i+1} \leq N_{i+1}<\ldots
$$

defined by the properties

$$
\begin{array}{ll}
A \subseteq \bigcup_{i}\left[M_{i}, N_{i}\right], & \left\{M_{i}, N_{i}\right\} \in A, \\
\left.g A \subseteq \bigcup_{i}\right] N_{i}, M_{i+1}\left[\cup \left[1, M_{1}[,\right.\right. & g A \cap] N_{i}, M_{i+1}[\neq \emptyset \quad \forall i .
\end{array}
$$

Since $\left.N_{i} \in A, g A \cap\right] N_{i}, M_{i+1}\left[\neq \emptyset\right.$ and $g A \subset B_{0}$, it follows that $T f\left(N_{i}\right)>\frac{2}{3}$ and $T f\left(N_{i}+k\right)<\frac{1}{3}$ for some $k<M_{i+1}-N_{i}$. From the estimate

$$
|T f(n)-T f(n+i)|=\left|\left(\frac{1}{n}-\frac{1}{n+i}\right) \sum_{j=1}^{n} f(j)-\frac{1}{n+i} \sum_{j=n+1}^{n+i} f(j)\right| \leq \frac{2 i\|f\|}{n+i}
$$

it follows that

$$
\frac{1}{3}<\left|T f\left(N_{i}\right)-T f\left(N_{i}+k\right)\right| \leq \frac{2 k\|f\|}{N_{i}+k}<\frac{2 k\|f\|}{N_{i}} .
$$

Therefore $k>N_{i} / 6\|f\|$ and we see that our sequences are lacunary, i.e.

$$
N_{i+1} \geq M_{i+1}>N_{i} q \geq M_{i} q, \quad 1<q=1+\frac{1}{6\|f\|} .
$$


Since $\left.g A \subset \bigcup_{i}\right] N_{i}, M_{i+1}\left[\right.$ we obtain the decomposition $A=A_{1} \cup A_{2}$ with

$$
\begin{aligned}
& A_{1}:=\{n \in A: g n<n\}=\bigcup_{i}\left\{n \in A \cap\left[M_{i}, N_{i}\right]: g n<M_{i}\right\}, \\
& A_{2}:=\{n \in A: g n>n\}=\bigcup_{i}\left\{n \in A \cap\left[M_{i}, N_{i}\right]: g n>N_{i}\right\} .
\end{aligned}
$$

It now suffices to show that both $A_{1}$ and $A_{2}$ are sets of density 0 .

For $M_{K} \leq N \leq M_{K+1}$ we have

$$
\frac{1}{N}\left|\left\{n \in A_{1}: n \leq N\right\}\right| \leq \frac{1}{M_{K}} \sum_{i=1}^{K} \phi_{i} M_{i},
$$

where $\phi_{i}=\left|\left\{n \in A \cap\left[M_{i}, N_{i}\right]: g n<M_{i}\right\}\right| / M_{i} \rightarrow 0$ as $i \rightarrow \infty$ since $g \in \mathcal{G}$. With the above lacunarity property $M_{i+1}>q M_{i}$ and $q>1$ we obtain by an argument similar to (5) in the proof of Lemma 4

$$
\lim _{K \rightarrow \infty} \frac{1}{M_{K}} \sum_{i=1}^{K} \phi_{i} M_{i} \leq \lim _{K \rightarrow \infty} \sum_{i=1}^{K} \phi_{i} q^{i-K}=0,
$$

which shows with (29) that $A_{1}$ has density 0 .

For $A_{2}$ and $N_{K-1} \leq M<N_{K}$ we have

$$
\frac{1}{N}\left|\left\{n \in A_{2}: n \leq N\right\}\right| \leq \frac{1}{N_{K-1}} \sum_{i=1}^{K-1} \phi_{i} N_{i}+r_{N},
$$

where $\phi_{i}=\left|\left\{n \in A \cap\left[M_{i}, N_{i}\right]: g n>N_{i}\right\}\right| / N_{i} \rightarrow 0$ and

$$
r_{N}=\frac{1}{N}\left|\left\{n \in A \cap\left[M_{K}, N\right]: g n>N_{K}\right\}\right| \leq \frac{1}{N} \mid\left\{n \in A \cap\left[M_{K}, N\right]: g n>N\right\} .
$$

Again since $g \in \mathcal{G}$ both $\phi_{i}$ and $r_{N}$ converge to 0 as $i$ respectively $N$ go to $\infty$. As above it follows that

$$
\lim _{K \rightarrow \infty} \frac{1}{N_{K-1}} \sum_{i=1}^{K-1} \phi_{i} N_{i} \leq \lim _{K \rightarrow \infty} \sum_{i=1}^{K-1} \phi_{i} q^{i-K+1}=0,
$$

and with (30) we see that $A_{2}$ has density 0 .

(v) $\Rightarrow$ (iii): If the $\mathcal{G}$-orbit of $x$ is dense in $\mathbb{N}^{*}$, then condition (v) implies that $T f$ is constant on $\mathbb{N}^{*}$, which is equivalent to $f$ being Cesàro-summable. Therefore $(\mathrm{v})$ can only hold for all $f$ in $C(\beta \mathbb{N})$ if the $\mathcal{G}$-orbit of $x$ is not dense.

(i) $\Rightarrow$ (vi): Assume $f$ is not constant on the $\mathcal{G}$-orbit of $x$, say $f(x)+3 \epsilon<f(g x)$ for some $\epsilon>0$ and $g \in \mathcal{G}$. Then the set $C:=\{n: f(n)<f(x)+\epsilon, f(g n)>f(g x)-\epsilon\}$ is contained in the ultrafilter $x$, so $C$ has positive upper density $\overline{d_{C}}$ since $x \in S$. But $\{n:|f(n)-f(g n)|>\epsilon\} \supset C$, so $f$ is not almost invariant.

(vi) $\Rightarrow$ (iii): If $A$ is an infinite subset of $\mathbb{N}$ with density 0 , it follows by Lemma 2 that $g A$ and therefore $A \triangle g A$ have density 0 , so $\chi_{\bar{A}}$ and $\chi_{\mathrm{C} \bar{A}}$ are almost invariant functions. Now (vi) implies $\mathcal{G} x \subset \bar{A}$ if $x \in \bar{A}$ and $\mathcal{G} x \subset \complement \bar{A}$ if $x \in \complement \bar{A}$. In either case the $\mathcal{G}$-orbit of $x$ is not dense in $\mathbb{N}^{*}$ since both $\bar{A}$ and $\complement \bar{A}=\bar{\complement} \bar{A}$ are open.

(i) $\Rightarrow$ (vii): Let $\bar{A}, A \subset \mathbb{N}$ be a neighborhood of $x$. Since $x \in S$ we have $\overline{d_{A}}>0$. By Theorem 3 there are $g_{1}, \ldots, g_{m} \in \mathcal{G}$ such that $B \subset \bigcup_{i=1}^{m} g_{i} A$ for an almost invariant set $B \subset \mathbb{N}$ with $\overline{d_{B}}>0 . \bar{B} \cap S$ is nonempty, compact and $\mathcal{G}$-invariant by Lemma 6 . A standard application of Zorn's lemma shows that any nonempty compact invariant set contains a minimal orbit closure (cf. [19], II 3.12 or [9], 
2.22), so it contains almost periodic points by Lemma 7. If $y \in \bar{B} \cap S$ is almost periodic, then it follows from Lemma 7 that $g y$ is almost periodic for $g \in \mathcal{G}$. Now for some $i$ the point $g_{i}^{-1} y$ is in $\bar{A}$ since $\bar{B} \subset \bigcup_{i=1}^{m} g_{i} \bar{A}$ and $y \in \bar{B}$. Hence each clopen neighborhood $\bar{A}$ of $x$ contains an almost periodic point. Since these neighborhoods are a base for the neighborhood filter of $x$ we are done.

(vii) $\Rightarrow$ (iii): If a point $y \in \mathbb{N}^{*}$ has dense $\mathcal{G}$-orbit, then the orbit closure of $y$ is not minimal since $S$ is a closed invariant proper subset of $\mathbb{N}^{*}$, so $y$ is not almost periodic by Lemma 7. By Lemma 6 and the equivalence of (i) and (iii) the set $S$ of points without dense $\mathcal{G}$-orbit is closed in $\beta \mathbb{N}$, so the closure of the set of almost periodic points is contained in $S$.

Theorem 5. $f \in \ell^{\infty}(\mathbb{N})$ is almost invariant iff its continuous extension to $\beta \mathbb{N}$ is constant on the $\mathcal{G}$-orbits in $S$.

Proof. Since (i) implies (vi) in Theorem 4 it remains to show that functions which are not almost invariant are not constant on some $\mathcal{G}$-orbit in $S$. If for some $\epsilon>0$ the set $\{n: f(n)<f(g n)-3 \epsilon\}$ has positive upper density, then for some $k \in \mathbb{Z}$ and $g \in \mathcal{G}$ the set $C:=\{n: f(n)<k \epsilon, f(g n)>(k+1) \epsilon\}$ has positive upper density. So $\bar{C} \cap S \neq \emptyset$ by Lemma 6 and we have $f(x) \leq k \epsilon, f(g x) \geq(k+1) \epsilon$ for $x \in \bar{C} \cap S$, so $f$ is not constant on the $\mathcal{G}$-orbit of $x \in \bar{C} \cap S$.

With Theorem 4 we therefore have:

Corollary 2. The function $T f$ is almost invariant $\forall f \in \ell^{\infty}(\mathbb{N})$.

For the dynamical system $(\tau, \beta \mathbb{N}), \tau$ being the shift operator, the support set of $\tau$-invariant measures was investigated in [17] and [4]. Lemma 1.3 in [4] gives a characterization using a different notion of upper density that strongly resembles the equivalence of (i) and (iv) in Theorem 4 above. Concerning the equivalence of (iv) and (vii) in Theorem 4 we remark that for the shift operator (or more generally certain amenable semigroup actions on the Stone-Čech compactification of the semigroup) there always exist open subsets in the support set of invariant measures, which do not contain almost periodic points cf. [4], [7]. So (iv) $\Rightarrow$ (vii) is false in the setting of the dynamical system $(\tau, \beta \mathbb{N})$.

\section{NON-MINIMAL ORBIT CLOSURES IN $S$}

In view of Theorem 4 it is natural to ask whether every point in $S$ is almost periodic, or equivalently, if all orbit closures in $S$ are minimal. We will show that there are non-minimal orbit closures in $S$ and that there are minimal orbit closures in $S$ which do not support any invariant measure. This property contrasts the case of amenable group actions on a compact space, where any closed nonempty invariant set supports an invariant measure cf. [19], IV 6.11.

We first define ultrafilters with non-minimal orbit closures: For $i, j, n=0,1, \ldots$ let

$$
\begin{gathered}
M_{j}:=\left\{2^{j} k: k \in \mathbb{N}\right\}, \quad J_{n, j}=\left[2^{\frac{n(n+1)}{2}+j}, 2^{\frac{n(n+1)}{2}+j+1}[,\right. \\
I_{n, j}:=J_{n, j} \cap M_{j}, \quad E_{i}:=\bigcup_{n=i}^{\infty} \bigcup_{j=i}^{n} I_{n, j},
\end{gathered}
$$


i.e. $E_{0}$ consists of successive intervals of doubling length with the "densities" in these intervals being $2^{0}, 2^{0}, 2^{-1}, 2^{0}, 2^{-1}, 2^{-2}, 2^{0}, 2^{-1}, 2^{-2}, 2^{-3}, \ldots$ respectively. $E_{i}$ then is just $E_{0}$ with all intervals with density greater than $2^{-i}$ being removed.

Clearly any set $E_{i}$ has positive upper density and $E_{i} \cap E_{j}=E_{j}$ for $j \geq i$. Together with the collection of all sets of density one, the sets $E_{i}$ define a filter base for a filter $\mathfrak{F}_{0}$ with all ultrafilters containing $\mathfrak{F}_{0}$ being in $S$.

Theorem 6. No ultrafilter containing $\mathfrak{F}_{0}$ is almost periodic with respect to $\mathcal{G}$.

Proof. Let $p$ be an ultrafilter containing $\mathfrak{F}_{0}$. To show that $p$ is not an almost periodic point of $\beta \mathbb{N}$, we have to show that for some neighborhood $U$ of $p$ the set $\{g: g p \in U\}$ is not syndetic. Our neighborhood will be $\overline{E_{0}}$. To prove that the set $\mathcal{A}:=\left\{g: g p \in \overline{E_{0}}\right\}$ is not syndetic, we have to find for any finite collection $g_{0}, g_{1}, \ldots, g_{m}$ of elements in $\mathcal{G}$ a $g^{\prime} \in \mathcal{G}$ with $g^{\prime} p \notin \bigcup_{j=0}^{m} g_{j} \mathcal{A} p$. As $\mathcal{A} p \subset \overline{E_{0}}$ this will follow, if we show that $g^{\prime} p \notin \bigcup_{j=0}^{m} g_{j} \overline{E_{0}}$.

We choose $i \in \mathbb{N}$ such that $2^{i}>m+1$ and define sets $A_{0}:=g_{0} E_{i}, A_{1}:=$ $g_{1} E_{i} \backslash g_{0} E_{i}, \ldots, A_{l}:=g_{l} E_{i} \backslash\left(\bigcup_{j=0}^{l-1} g_{j} E_{i}\right), 1 \leq l \leq m$, so $\bigcup_{l=0}^{m} g_{l} E_{i}$ is the disjoint union of the sets $A_{l}, l=0,1, \ldots, m$. It follows that

$$
d_{\bigcup_{l=0}^{m} g_{l} E_{i}}(n)=\sum_{l=0}^{m} d_{A_{l}}(n) \quad \text { for } n \in \mathbb{N} .
$$

We define for $0 \leq l \leq m$ sets $B_{l}:=\tau^{l} g_{l}^{-1} A_{l} \subset \tau^{l} E_{i}, \tau$ being the right shift operator $\tau C=\{n \in \mathbb{N}: n-1 \in C\}$ and $B:=\bigcup_{l=0}^{m} B_{l} \subset \bigcup_{l=0}^{m} \tau^{l} E_{i}$. Now since $d_{\tau^{l} C}(n)=$ $\frac{n-l}{n} d_{C}(n-l)$, for $C \subset \mathbb{N}$, it follows with Lemma 2 , that $\lim _{n \rightarrow \infty} d_{B_{l}}(n)-d_{A_{l}}(n)=0$. Since the sets $\tau^{l} E_{i}$, are pairwise disjoint for $l=0, \ldots, m$ by our choice of $i$, so are the sets $B_{l}$, hence $d_{B}(n)=\sum_{l=0}^{m} d_{B_{l}}(n)$ and with (32) we obtain

$$
\lim _{n \rightarrow \infty} d_{B}(n)-d_{\cup_{j} g_{j} E_{i}}(n)=\sum_{l=0}^{m} \lim _{n \rightarrow \infty} d_{B_{l}}(n)-d_{A_{l}}(n)=0 .
$$

By Lemma 3 there exists $g \in \mathcal{G}$ with $g B=\bigcup_{j=0}^{m} g_{j} E_{i}$. Since $B \subset \bigcup_{l=0}^{m} \tau^{l} E_{i}$ we have $\left(\tau^{m+1} E_{i}\right) \cap B=\emptyset$ and $\left(g \tau^{m+1} E_{i}\right) \cap\left(\bigcup_{j=0}^{m} g_{j} E_{i}\right)=\emptyset$. Applying Lemma 3 again, we see that $\tau^{m+1} E_{i}=\tilde{g} E_{i}, \tilde{g} \in \mathcal{G}$, so $g \tau^{m+1} E_{i}=g^{\prime} E_{i}$ for some $g^{\prime} \in \mathcal{G}$ and $\bigcup_{j=0}^{m} g_{j} E_{i} \cap g^{\prime} E_{i}=\emptyset$, hence $\bigcup_{j=0}^{m} g_{j} \overline{E_{i}} \cap g^{\prime} \overline{E_{i}}=\emptyset$. Since $p \in \overline{E_{i}}$ it follows that $g^{\prime} p \notin \bigcup_{j=0}^{m} g_{j} \overline{E_{i}}$.

We define with (31) a set $F_{i}:=\bigcup_{k=0}^{\infty} \bigcup_{l=0}^{i-1} J_{k, l}$. By Lemma $4 F_{i}$ is almost invariant. Since $E_{i} \cap F_{i}=\emptyset$, it follows that $p \notin \overline{F_{i}}$, and since $p \in S$, we conclude with Lemma 6 that $g^{\prime} p \notin \overline{F_{i}}$. But $E_{0} \subset F_{i} \cup E_{i}$, so $\bigcup g_{j} \overline{E_{0}} \cap S \subset\left(\overline{F_{i}} \cup g_{j} \overline{E_{i}}\right) \cap S$. Since we have shown that $g^{\prime} p \in S$ is neither in $\bigcup_{j=0}^{m} g_{j} \overline{E_{i}}$ nor in $\overline{F_{i}}$, it follows that $g^{\prime} p \notin \bigcup_{j=0}^{m} g_{j} \overline{E_{0}}$.

Theorem 7. No invariant measure is supported in the $\mathcal{G}$-orbit closure of any ultrafilter $p$ containing $\mathfrak{F}_{0}$. Therefore there exist minimal $\mathcal{G}$-orbit closures in $S$ which do not support any $\mathcal{G}$-invariant measure.

Proof. It is sufficient to consider probability measures only since the positive part $\mu_{+}$of an invariant measure $\mu$ is invariant (cf. (3) in the proof of Lemma 1). So let $\mu$ be a $\mathcal{G}$-invariant probability measure supported in $\overline{\mathcal{G} p}$. By Lemma 4 the set $D_{i}:=\bigcup_{n=0}^{\infty} \bigcup_{j=i}^{n} J_{n, j}$ is almost invariant for $i \in \mathbb{N}$. Clearly $D_{i} \in \mathfrak{F}_{0}$, so $p \in \overline{D_{i}} \cap S$ 
and $\overline{\mathcal{G} p} \subseteq \overline{D_{i}}$ by Theorem 4 since $\chi_{\overline{D_{i}}}$ is an almost invariant function and $p \in S$. Then $\mu\left(\overline{E_{0}}\right)=\mu\left(\overline{E_{i}}\right)$ since $\overline{E_{0}} \backslash \overline{E_{i}}$ is disjoint from $\overline{D_{i}}$, and hence disjoint from $\overline{\mathcal{G} p}$. As the permutation $g_{0}$ defined by $g_{0} n=n+1$ for $n \neq 2^{l}-1, g_{0}\left(2^{l}-1\right)=2^{l-1}$, $l \geq 1$, is in $\mathcal{G}$ and the sets $g_{0}^{k} \overline{E_{i}}, k=0, \ldots, 2^{i}-1$ are pairwise disjoint, it follows that $\mu\left(\overline{E_{0}}\right)=\mu\left(\overline{E_{i}}\right)<2^{-i}$. Since $i$ was arbitrary, it follows that $\mu\left(\overline{E_{0}}\right)=0$ and $p$ is not in the support of $\mu$ as $p \in \overline{E_{0}}$.

The second statement follows since every orbit closure on a compact space contains a point with minimal orbit closure by Zorn's lemma.

Corollary 3. $\mathcal{G}$ is not amenable.

Proof. Amenability is equivalent to the existence of invariant means on any compact $\mathcal{G}$-space cf. [19], IV 6.11.

\section{$T^{*}$-INVARIANT MEASURES}

It is easily seen that the measure $\delta_{1}$ is the only $T^{*}$-invariant probability measure supported in $\mathbb{N}$. Let

$$
\mathcal{M}_{I}=\left\{\mu \in \mathcal{M}(\beta \mathbb{N}):\langle\mu, f\rangle=\left\langle T^{*} \mu, f\right\rangle=\langle\mu, T f\rangle \quad \forall f \in C(\beta \mathbb{N})\right\} \cap \mathcal{M}_{\infty}
$$

be the space of $T^{*}$-invariant measures in $\mathcal{M}_{\infty}$ (space of measures supported in $\mathbb{N}^{*}$ ). Clearly $\mathcal{M}_{I}$ is a weak ${ }^{*}$-closed subspace of $\mathcal{M}$. Since weak and norm-closure of a linear subspace coincide (cf. [6], V.3.13), it follows from the bipolar theorem [18], III.7.4, that

$$
\mathcal{M}_{I}^{\perp}=\left(\operatorname{Span}\left(\operatorname{Ran}(I-T) \cup\left\{e_{i}\right\}\right)\right)^{\perp \perp}=\overline{\operatorname{Span}\left(\operatorname{Ran}(I-T) \cup\left\{e_{i}\right\}\right)}
$$

(norm-closure), where $e_{1} \in C(\beta \mathbb{N})$ is given by $e_{1}(1)=1, e_{1}(n)=0, n>1$. The support of any measure in $\mathcal{M}_{I}$ is disjoint from $\mathbb{N}$, so it follows from Theorem 2 that $\mathcal{M}_{I} \subseteq \mathcal{M}_{\mathcal{G}}$.

We denote the set of probability measures in $\mathcal{M}_{\infty}$ by $\mathcal{P}$. As $T^{*}$ maps $\mathcal{P}$ to $\mathcal{P}$, the compact convex set $\mathcal{P}$ has a fixed point under the map $T^{*}$ by the Markov-Kakutani fixed point theorem, and $\mathcal{M}_{I}$ is not empty. We can moreover give an explicit description (similar to Theorem 2) of a subset of $\mathcal{M}_{I}$ which has a weak*-dense linear hull in $\mathcal{M}_{I}$ :

Let

$$
\mathcal{L}_{I}=\left\{f \in C(\beta \mathbb{N}): \lim _{N \rightarrow \infty} \frac{1}{N} \sum_{n=1}^{N} T^{n} f(p)=0 \quad \forall p \in \mathbb{N}^{*}\right\} .
$$

Clearly $\mathcal{L}_{I}$ is a norm (and therefore weakly) closed subspace of $C(\beta \mathbb{N})$ containing $\mathcal{L}_{0}$ (as defined in the proof of Theorem 2). For arbitrary $f$ in $C(\beta \mathbb{N})$ the limit

$$
\lim _{N \rightarrow \infty} \frac{1}{N} \sum_{n=1}^{N} T^{n} f(p)
$$

need not exist for all $p$. For fixed $p \in \beta \mathbb{N}$ the sequence $\left(c_{N}(p)\right)_{N=1}^{\infty}$ with $c_{N}(p):=$ $\frac{1}{N} \sum_{n=1}^{N} T^{n} f(p)$ can be considered as an element of $\ell^{\infty}(\mathbb{N})$. The continuous extension to $\beta \mathbb{N}$ can be evaluated at a point $q$ of $\beta \mathbb{N}$. In order to distinguish between our phase space $\beta \mathbb{N}$ and this functional on $\ell^{\infty}(\mathbb{N})$ we shall use Z. Frolík's notation $q-\lim _{N \in \mathbb{N}} c_{N}(p)$ for the evaluation of the continuous extension of $\left(c_{N}(p)\right)$ to $\beta \mathbb{N}$ at the point $q$. If $p, q \in \mathbb{N}^{*}$ and $f \in C(\beta \mathbb{N})$ then $q-\lim _{N \in \mathbb{N}} \frac{1}{N} \sum_{n=1}^{N} T^{n} f(p)$ is a positive linear functional of norm 1 on $C(\beta \mathbb{N})$, which we denote by $\nu_{p, q}$. 
It is easily seen that $\nu_{p, q}$ is a $T^{*}$-invariant probability measure. We now show that the linear hull $\mathcal{H}$ of the set $\left\{\nu_{p, q}: p, q \in \mathbb{N}^{*}\right\}$ is weak ${ }^{*}$-dense in $\mathcal{M}_{I}$ :

Let $f \in \mathcal{H}^{\perp}$, i.e.

$$
q-\lim _{N \in \mathbb{N}} \frac{1}{N} \sum_{n=1}^{N} T^{n} f(p)=0 \quad \forall p, q \in \mathbb{N}^{*}
$$

then

$$
\lim _{N \rightarrow \infty} \frac{1}{N} \sum_{n=1}^{N} T^{n} f(p)=0 \quad \forall p \in \mathbb{N}^{*},
$$

so $f \in \mathcal{L}_{I}$. Therefore $\mathcal{H}^{\perp} \subseteq \mathcal{L}_{I}$.

On the other hand, for $\mu \in \mathcal{M}_{I}$ and $f \in \mathcal{L}_{I}$, we obtain

$$
\langle\mu, f\rangle=\left\langle T^{*} \mu, f\right\rangle=\frac{1}{N}\left\langle\sum_{n=1}^{N} T^{* n} \mu, f\right\rangle=\left\langle\mu, \frac{1}{N} \sum_{n=1}^{N} T^{n} f\right\rangle,
$$

which by Lebesgue's convergence theorem yields $\langle\mu, f\rangle=\langle\mu, 0\rangle=0$, so $\mathcal{M}_{I}^{\perp} \supseteq \mathcal{L}_{I}$. Since $\mathcal{M}_{I} \supseteq \mathcal{H}$ we have $\mathcal{M}_{I}^{\perp} \subseteq \mathcal{H}^{\perp}$, so we obtain

$$
\mathcal{H}^{\perp} \subseteq \mathcal{L}_{I} \subseteq \mathcal{M}_{I}^{\perp} \subseteq \mathcal{H}^{\perp}
$$

and it follows that $\mathcal{H}^{\perp \perp}=\mathcal{M}_{I}^{\perp \perp}$. Again by the bipolar theorem we see that $\mathcal{H}^{\perp \perp}$ is the weak ${ }^{*}$-closure of $\mathcal{H}$ and $\mathcal{M}_{I}^{\perp \perp}=\mathcal{M}_{I}$, since $\mathcal{M}_{I}$ being the annihilator of $\operatorname{Ran}(I-T)$ is weak*-closed. With (33) we thus have shown:

Theorem 8. $\mathcal{M}_{I}$ is a weak ${ }^{*}$-closed subspace of $\mathcal{M}_{\mathcal{G}}$ with annihilator $\mathcal{M}_{I}^{\perp}=\mathcal{L}_{I}=$ $\overline{\operatorname{Span}\left(\operatorname{Ran}(I-T) \cup\left\{e_{i}\right\}\right)} . \mathcal{H}$ is weak ${ }^{*}$-dense in $\mathcal{M}_{I}$.

We now determine the support set of measures in $\mathcal{M}_{I}$ and get a similar characterization as the equivalence of (i) and (iv) in Theorem 4 :

Corollary 4. If for $A \subset \mathbb{N}$ we have $\left\langle\nu_{p, q}, \chi_{\bar{A}}\right\rangle=0 \forall p, q \in \mathbb{N}^{*}$, then $\bar{A}$ is disjoint from the support set of $\mathcal{M}_{I}$.

Proof. Since $\nu_{p, q}$ is a probability measure and $\chi_{\bar{A}}$ is positive, it follows that the support of $\nu_{p, q}$ does not intersect $\bar{A}$ for $p, q \in \mathbb{N}^{*}$. Therefore any continuous function on $\beta \mathbb{N}$ vanishing outside of $\bar{A}$ is in $\mathcal{H}^{\perp}$. But $\mathcal{H}^{\perp}=\mathcal{M}_{I}^{\perp}$ by Theorem 8 or (34), so the open set $\bar{A}$ is disjoint from the support of any measure in $\mathcal{M}_{I}$, and the claim follows.

Let $S_{I}$ be the set of points $x$ in $\beta \mathbb{N}$ with the property that no clopen neighborhood $\bar{A}, A \subset \mathbb{N}$, of $x$ satisfies

$$
\lim _{N \rightarrow \infty} \frac{1}{N} \sum_{n=1}^{N} T^{n} \chi_{\bar{A}}(p)=0 \quad \forall p \in \mathbb{N}^{*}
$$

i.e. for no clopen neighborhood $\bar{A}, A \subset \mathbb{N}$, of $x$ is the function $\chi_{\bar{A}}$ in $\mathcal{L}_{I}$.

Theorem 9. $S_{I}$ is the support set of $\mathcal{M}_{I}$.

Proof. If $x \notin S_{I}$, then there exists a clopen neighborhood $\bar{A}$ of $x$ with

$$
\lim _{N \rightarrow \infty} \frac{1}{N} \sum_{n=1}^{N} T^{n} \chi_{\bar{A}}(p)=0
$$


for all $p \in \mathbb{N}^{*}$, therefore $\left\langle\nu_{p, q}, \chi_{\bar{A}}\right\rangle=0 \quad \forall p, q \in \mathbb{N}^{*}$. It follows from Corollary 4 that $x$ is not in the support set of $\mathcal{M}_{I}$.

If $x \in S_{I}$, then for any clopen neighborhood $\bar{A}$ of $x$, there exists a measure $\nu_{p, q}$, such that $\left\langle\nu_{p, q}, \chi_{\bar{A}}\right\rangle \neq 0$, i.e. $\bar{A}$ intersects the support of $\nu_{p, q}$ for some $p, q \in \mathbb{N}^{*}$. Since the clopen sets containing $x$ are a neighborhood base of $x$ it follows that $x$ is in the support set of $\mathcal{M}_{I}$.

Theorem 10. $S_{I}$ is a nonempty proper closed subset of $S$.

Proof. Clearly $S_{I} \subseteq S$ and $S_{I}$ is closed and nonempty by Theorem 9, as support sets are by definition closed and nonempty if invariant measures exist.

To prove that $S_{I} \neq S$ we show that there exists a subset $A$ of $\mathbb{N} \backslash\{1\}$ with $\overline{d_{A}}>0$ such that $\chi_{\bar{A}} \in \overline{\operatorname{Ran}(I-T)}$ (norm-closure): By Lemma $6 \bar{A} \cap S \neq \emptyset$, but by Theorem $8 \chi_{\bar{A}} \in \mathcal{M}_{I}^{\perp}$, so Corollary 4 with Theorem 9 show that $\bar{A} \cap S_{I}=\emptyset$, hence $S_{I} \neq S$.

For any $A \subset \mathbb{N} \backslash\{1\}$ we can define inductively a function $F$ with $(I-T) F(n)=$ $\chi_{A}(n)$. However $F$ will not be bounded in general, since $F$ must be constant on $\complement A$ and increasing on $A$ (see the computations below). We therefore define the function $f$ on $\mathbb{N}$, such that $f$ decreases on $\complement A$ in order to guarantee boundedness, but still satisfies $f-T f-\chi_{A} \in c_{0}$. Now $g \in c_{0}(\mathbb{N})$ is in $\overline{\operatorname{Ran}(I-T)}$ by Theorem 8 if $g(1)=0$, so this will be sufficient to show that $\chi_{\bar{A}} \in \overline{\operatorname{Ran}(I-T)}$.

The set $A$ will be $\bigcup_{i}\left[N_{i}, 2 N_{i}\right]$ for a suitably chosen sequence $\left(N_{i}\right)$. Clearly $A$ has upper density $\overline{d_{A}} \geq 1 / 2$, so $\bar{A}$ intersects $S$ by Lemma 6 .

Let $N_{1}=3$ and $f(1)=f(2)=0$. Assume $N_{1}, \ldots, N_{n}$ have been chosen and $f$ has been defined on $[1, l-1]$ for some $l, N_{n} \leq l \leq 2 N_{n}$. We now define $f(l)$ by

$$
f(l):=T f(l-1)+\frac{l}{l-1},
$$

and obtain

$$
\begin{aligned}
f(l)-T f(l) & =\frac{l-1}{l} f(l)-\frac{1}{l} \sum_{j=1}^{l-1} f(j) \\
& =\frac{l-1}{l}(f(l)-T f(l-1))=\frac{l-1}{l} \frac{l}{l-1}=1 .
\end{aligned}
$$

For $N_{n}<l \leq 2 N_{n}$ this yields

$$
\begin{aligned}
f(l) & =T f(l-1)+\frac{l}{l-1} \\
& =f(l-1)-1+\frac{l}{l-1}=f(l-1)+\frac{1}{l-1} .
\end{aligned}
$$

Therefore $f$ is increasing in $\left[N_{n}, 2 N_{n}\right]$ and we obtain

$$
\begin{aligned}
f\left(2 N_{n}\right) & =f\left(N_{n}\right)+\sum_{l=N_{n}}^{2 N_{n}-1} \frac{1}{l} \leq f\left(N_{n}\right)+\log \left(2 N_{n}-1\right)-\log \left(N_{n}-1\right) \\
& \leq f\left(N_{n}\right)+2 .
\end{aligned}
$$

For $l>2 N_{n}$ and $f(l-1)>\frac{1}{n}$ we define

$$
f(l):=T f(l-1)-\frac{l}{l-1} \frac{1}{n},
$$


and obtain

$$
f(l)-T f(l)=\frac{l-1}{l}(f(l)-T f(l-1))=-\frac{l-1}{l} \frac{1}{n} \frac{l}{l-1}=-\frac{1}{n} .
$$

For $l=2 N_{n}+1$ we have with (38), (36)

$$
f\left(2 N_{n}+1\right)=T f\left(2 N_{n}\right)-\frac{2 N_{n}+1}{2 N_{n}} \frac{1}{n}=f\left(2 N_{n}\right)-1-\frac{2 N_{n}+1}{2 N_{n}} \frac{1}{n},
$$

and for $l>2 N_{n}+1, f(l-1)>\frac{1}{n}(38)$ and (39) yield

$$
f(l)=T f(l-1)-\frac{l}{l-1} \frac{1}{n}=f(l-1)+\frac{1}{n}-\frac{l}{l-1} \frac{1}{n}=f(l-1)-\frac{1}{n} \frac{1}{l-1}>0 .
$$

Therefore there exists a smallest $l_{n}>N_{n}$ such that $f\left(l_{n}-1\right) \leq \frac{1}{n}$ and we set $N_{n+1}:=l_{n}$ and continue by defining $f$ on $\left[N_{n+1}, 2 N_{n+1}\right]$ as above.

The function $f$ so constructed satisfies $(f-T f)(l)=1$ on $A=\bigcup_{i}\left[N_{n}, 2 N_{n}\right]$ by (36) and $|(f-T f)(l)|=\frac{1}{n}$ on the intervals $] 2 N_{n}, N_{n+1}$ [ by (39). It follows that $f-T f-\chi_{A} \in c_{0}$.

We have $f\left(N_{n+1}-1\right)<\frac{1}{n}$ by our choice of $N_{n+1}$, hence

$$
T f\left(N_{n+1}-1\right)-\frac{1}{n}=f\left(N_{n+1}-1\right)<\frac{1}{n}
$$

by (39), so

$$
f\left(N_{n+1}\right)=T f\left(N_{n+1}-1\right)+\frac{N_{n+1}}{N_{n+1}-1}<\frac{2}{n}+\frac{N_{n+1}}{N_{n+1}-1}<4
$$

by (35) and $f\left(2 N_{n+1}\right)<6$ by (37). Since $f$ is increasing on $\left[N_{n}, 2 N_{n}\right]$ and decreasing on $\left[2 N_{n}, N_{n+1}\right.$ [ by (40) and (37), it follows with (41) that $0 \leq f(n)<6 \forall n \in \mathbb{N}$, i.e. $f \in \ell^{\infty}(\mathbb{N})$.

\section{REFERENCES}

1. Bergelson V., Hindman N., Nonmetrizable topological dynamics and Ramsey theory, Trans. Amer. Math. Soc. 320 (1990), 293-320. MR 90k:03046

2. Berglund J.F., Junghenn H.D., Milnes P., Compact right topological semigroups and generalizations of almost periodicity, Springer, LNM 663, Berlin, 1978. MR 80c:22003

3. Blümlinger M., Obata N., Permutations preserving Cesàro mean, densities of natural numbers and uniform distribution of sequences, Ann. Inst. Fourier 41 (1991), 665-678. MR 92j:43002

4. Chou C., Minimal sets and ergodic measures for $\beta \mathbb{N} \backslash \mathbb{N}$, Illinois J. Math. 13 (1969), 777-788. MR 40:2814

5. van Douwen E., The Čech-Stone compactification of a discrete groupoid, Topology Appl. 39 (1991), 43-60. MR 92g:54026

6. Dunford N., Schwartz J., Linear Operators I, Wiley, New York, 1957. MR 22:8302

7. Fairchild L., Extreme invariant means without minimal support, Trans. Amer. Math. Soc. 172 (1972), 83-93. MR 46:7799

8. Furstenberg H., Recurrence in Ergodic Theory and Combinatorial Number Theory, Princeton Univ. Press, Princeton, 1981. MR 82j:28010

9. Gottschalk W.H., Hedlund G.H., Topological Dynamics, AMS, Providence, 1955. MR 17:650e

10. Hindman N., Ultrafilters and combinatorial number theory (M. Nathanson, ed.), LNM $\mathbf{7 5 1}$ (1979). MR 81m:10019

11. Hewitt E., Stromberg K., Real and Abstract Analysis, Springer, Berlin, 1965. MR 32:5826

12. Jerison M., The set of all generalized limits of bounded sequences, Canad. Math. Jour. 9 (1957), 79-89. MR 18:747g

13. Lévy P., Problèmes Concrets d'Analyse Fonctionnelle, Gauthier-Villars, Paris, 1951. MR 12: $834 \mathrm{a}$ 
14. Obata N., A note on certain permutation groups in the infinite dimensional rotation group, Nagoya Math. J. 109 (1988), 91-107. MR 89c:11020

15. _ Density of natural numbers and the Lévy group, J. Number Theory 30 (1988), 288297. MR 90e: 11027

16. Raimi R., Invariant means and invariant matrix methods of summability, Duke Math. Jour. 30 (1963), 81-91. MR 27:3965

17. 1-12. MR 33:6608

18. Taylor A., Lay D., Introduction to Functional Analysis, Wiley, New York, 1980. MR 81b:46001

19. de Vries J., Elements of Topological Dynamics, Kluwer, Dordrecht, 1993. MR 94m:54098

20. Walker R., The Stone-Čech Compactification, Springer, Berlin, 1974. MR 52:1595

Institut 114, Technische Universität Wien, Wiedner Hauptstr. 8-10, 1040 Wien, AusTRIA

E-mail address: mbluemli@email.tuwien.ac.at 